\title{
On the creation of the chromospheres of solar type stars
}

\author{
M. L. Goodman ${ }^{\star}$ \\ Institute for Scientific Research, 2500 Fairmont Avenue - Suite 734, Fairmont, WV 26555-2720, USA \\ e-mail: mgoodman@isr.us
}

Received 20 February 2004 / Accepted 19 April 2004

\begin{abstract}
A mechanism that creates the chromospheres of solar type stars everywhere outside of flaring regions is proposed. The identification of the mechanism is based on previous work and on the results of a model presented here that computes the electric current, its driving electric field, the heating rate due to resistive dissipation, and the flow velocity in a specified class of horizontally localized, two dimensional magnetic structures in the steady state approximation. The model is applied to the Sun over the height range from the photosphere to the upper chromosphere. Although the model does not contain time explicitly, it contains information about the dynamics of the atmosphere through inputs from the FAL CM solar atmosphere model, which is based on time averages of spectroscopic data. The model is proposed to describe the time averaged properties of the heating mechanism that creates the chromosphere. The model magnetic structure is horizontally localized, but describes heating of the global chromosphere in the following way. Recent observations indicate that kilogauss strength magnetic structures exist in the photospheric internetwork with a filling factor $f \sim 2 \%$, and characteristic diameters $<180 \mathrm{~km}$. Assuming $f=2 \%$ and a maximum field strength of $10^{3} \mathrm{G}$ for the model magnetic structure, and assuming that the chromospheric heating rate predicted by FAL CM represents a horizontal spatial average over such magnetic structures, it is found that the model magnetic structures that best reproduce the FAL CM heating rate as a function of height have characteristic diameters in the range of 98-161 km, consistent with the upper bound inferred from observation. Based on model solutions and previous work it is proposed that essentially all chromospheric heating occurs in magnetic structures with sub-resolution horizontal spatial scales $(\$ 150 \mathrm{~km})$, that the heating is due to dissipation of Pedersen currents driven by a convection electric field, and that it is the increase in the magnetization of particles with height in a magnetic structure from values $\ll 1$ in the lower photosphere to values $\gtrsim 1$ near the height of the temperature minimum in the magnetic structure that causes the Pedersen current dissipation rate to increase to a value large enough to cause a temperature inversion. The magnetization of a particle is the ratio of its cyclotron frequency to its total collision frequency with unlike particle species.
\end{abstract}

Key words. Sun: chromosphere - Sun: photosphere - magnetohydrodynamics (MHD) - Sun: magnetic fields stars: chromospheres - magnetic fields

\section{Introduction}

Convection of plasma exists everywhere and always over a wide range of temporal and spatial scales in the solar atmosphere, and presumably in the atmospheres of all solar type stars. A magnetic field $\boldsymbol{B}$ in the presence of convection provides a mechanism for the conversion of kinetic energy of convection into the electrical energy of a convection electric field $\boldsymbol{E}_{\mathrm{c}}$ that drives Pedersen current that is dissipated by collisions, generating thermal energy (Goodman 2000, 2001). $\boldsymbol{E}_{\mathrm{c}}=(\boldsymbol{V} \times \boldsymbol{B}) / c$ where $\boldsymbol{V}$ is the center of mass (CM) velocity of the plasma and $c$ is the speed of light. The atmosphere is a magnetohydrodynamic (MHD) power generator, converting mechanical energy of convection into electrical energy that is dissipated by the resistive load of the plasma. The magnetic field enables and mediates this energy conversion through the convection electric field. This heating mechanism operates to some degree across

^ Also with the Department of Physics, The Catholic University of America, Washington, DC, USA. the full range of length and time scales that characterize the magnetic field and convection in the atmosphere.

The specific convective processes that are the primary drivers of this heating mechanism need to be identified. The work presented here and by Goodman $(2000,2001)$ indicates that in order for this heating mechanism to balance the net radiative loss from the chromosphere it must be concentrated in horizontally localized magnetic structures with sub-resolution horizontal scales. A sub-resolution structure is defined as one with a characteristic diameter $D \lesssim 0.2^{\prime \prime} \sim 150 \mathrm{~km}$. This definition follows from the highest current observational resolution of bright points associated with kilogauss magnetic field concentrations in the photosphere (Lites et al. 1999). Goodman (2000, 2001) identifies MHD wave driven convection in such magnetic structures as a primary driver. All convection is time dependent, so a determination of the importance of other convective processes in driving the heating mechanism requires time dependent models, which are not considered here. Here only a few general remarks are made about driving mechanisms 
other than MHD waves. (i) Granular convection on space and time scales of the granular diameter of $\sim 10^{3} \mathrm{~km}$, and the granular turnover time of 5-10 min generates a convection electric field in magnetic structures concentrated in the boundary regions of the granulation. Here it is proposed that chromospheric heating occurs in magnetic structures with horizontal diameters $\sim 100 \mathrm{~km}$. If most of these structures have photospheric field strengths $\sim 10^{3} \mathrm{G}$, and a filling factor $\sim 0.01$, then the average separation between these structures is $\sim 10^{3} \mathrm{~km}$, which is the characteristic diameter of a granule. (ii) Horizontally localized magnetic structures in the solar atmosphere can exist only in the presence of a localized current density that contributes to heating through resistive dissipation. This current is independent of any MHD wave driven current that is generated in these structures, and might be a major source of heating. The current may be driven by a convection electric field.

Here a model is used to describe the time averaged effects of resistive dissipation in magnetic structures in the photosphere and chromosphere due to the collective effect of whatever mechanisms drive it. The model shows how the increase with height of the magnetization of charged particles, beginning in the photosphere, can cause a temperature inversion and create the chromospheres of the Sun and other solar type stars everywhere outside of flaring regions. The magnetization $M_{s}$ of a particle species $s$ is the ratio of its cyclotron frequency to its total collision frequency with unlike particle species.

The model is a two dimensional, steady state MHD model of a horizontally localized magnetic structure that extends vertically from the photosphere through the upper chromosphere, and that includes an expression for the electrical conductivity tensor of the multi-species, variably ionized atmosphere. A specified photospheric filling factor is used to estimate the global resistive heating rate due to a uniform, horizontal distribution of these structures over the photosphere. The heating rate is due to dissipation of Pedersen and magnetic field aligned (parallel) currents. In Sect. 4 the heating rate is required to optimally match that of model CM of Fontenla, Avrett \& Loeser (communicated by Avrett \& Loeser (2001), and henceforth FAL CM) over the height range of the chromosphere (FAL CM is not to be confused with $\mathrm{CM}$ - center of mass). The resulting magnetic structures are found to have sub-resolution horizontal length scales, and the heating is found to be due almost entirely to Pedersen current dissipation driven by a convection electric field. The model is steady state in that time does not appear explicitly in the model. Information about the dynamics of the atmosphere enters the model implicitly through density and temperature profiles generated by FAL CM. These profiles are computed using time averages of spectroscopic data, and are inputs to the model used here. FAL CM is essentially the model used by Fontenla et al. (2002) to describe energy balance, bulk flow, and diffusion in the solar transition region. Here only that part of the model that describes the photosphere and chromosphere is used.

\section{The model - Part 1}

CGS units are used throughout the paper unless indicated otherwise. Cylindrical coordinates $(R, \theta, z)$ are used. All quantities are assumed to be independent of $\theta$ and time. $z$ labels height. $z=0$ labels optical depth unity in the FAL CM photosphere. The magnitude of any vector function $\boldsymbol{F}$ is denoted by $F$.

A specified two dimensional magnetic field is combined with an Ohm's law and data from FAL CM to compute the properties of anisotropic resistive heating in the photosphere and chromosphere. The electrical conductivity tensor, heating rates per unit volume due to parallel and Pedersen current dissipation, center of mass (CM) electric field that drives the current, and particle current densities and magnetizations are computed as functions of $R$ and $z$ from $z=0$ to the upper chromospheric height of $z_{\max }=2164.4 \mathrm{~km}$ where the FAL CM temperature $T=9983 \mathrm{~K}$.

The magnetization $M_{s}$ of a particle species $s$ is defined as

$$
\begin{aligned}
& M_{s}=\frac{\omega_{s}}{v_{s}^{*}} \\
& \omega_{s}=\frac{\left|q_{s}\right| B}{m_{s} c} .
\end{aligned}
$$

$\omega_{s}, q_{s}, m_{s}$, and $v_{s}^{*}$ are the cyclotron frequency, charge, and mass of a particle of species $s$, and the total reduced collision frequency of a particle of species $s$ with unlike particle species. $v_{s}^{*}$ is defined in Sect. 2.5.1.

The model is not extended above $z_{\max }$ for the following reason. The assumed magnetic field, defined in Sect. 2.1, has the following properties. It is closed in that all field lines return to the photosphere. It is strongly localized in the radial direction at all heights. Its $z$ dependence is given by the factor $\exp \left(-z / L_{B}\right)$, where $L_{B}$ is an assumed constant scale height. This $z$ dependence is assumed to be a sufficiently good approximation below the transition region for making order of magnitude estimates of heating rates. The assumed height dependence is unacceptable at greater heights where large temperature gradients in the transition region, and temperatures $\sim 10^{5}-10^{6} \mathrm{~K}$ in the transition region and corona are expected to rapidly and greatly increase the characteristic magnetic field scale height with increasing height. A magnetic field model that is also valid above the chromosphere must describe fields that undergo horizontal expansion with increasing height, that may have open field lines, and that have a magnitude that decreases with height in a way that is not reasonably well described by an exponential with a constant scale height.

The photosphere and chromosphere consist almost entirely of electrons, protons, singly ionized metallic ions, $\mathrm{H}$, He I, He II, and He III. An approximate conductivity tensor of this multi-species plasma is computed in Sect. 2.5 by treating the plasma as a three species plasma of electrons, a singly charged proxy ion that collectively represents protons and metallic ions, and a proxy neutral species that collectively represents $\mathrm{H}$ and $\mathrm{He} \mathrm{I}$.

\subsection{Magnetic field}

The $z$ component of the field is chosen to be

$B_{z}=B_{0}\left(1-\frac{R^{2}}{R_{0}^{2}}\right) \exp \left(-R^{2} / R_{0}^{2}\right) \exp \left(-z / L_{B}\right)$. 
The parameters $B_{0}, R_{0}$, and $L_{B}$ are the maximum field strength, core radius, and scale height. These parameters are to be specified.

For this choice of $B_{z}$ the magnetic flux through the any surface $z=$ constant is zero since $\int_{0}^{\infty} B_{z}(R, z) R \mathrm{~d} R=0$. All field lines are closed. The field is unipolar for $R<R_{0}$, and for $R>R_{0}$ with the opposite sign. The magnetic flux through the core region $R \leq R_{0}$ is $F_{0}=A_{0} B_{0} \exp (-1)$, where $A_{0}=\pi R_{0}^{2}$ is the area of the core region. The flux through the area with $R=2 R_{0}$ is $0.2 F_{0}$, so at this radius $80 \%$ of the core flux has returned to the photosphere.

Then $\nabla \cdot \boldsymbol{B}=0$ implies that

$B_{R}=\frac{B_{0} R}{2 L_{B}} \exp \left(-R^{2} / R_{0}^{2}\right) \exp \left(-z / L_{B}\right)$.

$B_{\theta}$ is determined by the $\theta$ component of the momentum equation, Eq. (53), that is solved in Sect. 5.1. This component is

$(\boldsymbol{J} \times \boldsymbol{B})_{\theta}=0$,

where $\boldsymbol{J}$ is the current density. Solving Eq. (5) for $B_{\theta}$ gives

$B_{\theta}=\tau B_{R}$

$\tau$ is a constant parameter that measures the twist of the magnetic field in any horizontal plane.

This choice for $\boldsymbol{B}$ is made more realistic by requiring that

$\frac{\partial B}{\partial R} \leq 0$.

This constraint ensures that $B_{0}$ is the maximum field strength. It is satisfied if and only if

$\frac{2\left(2-3^{1 / 2}\right)^{1 / 2} L_{B}}{\left(1+\tau^{2}\right)^{1 / 2}} \leq R_{0} \leq \frac{4 L_{B}}{\left(1+\tau^{2}\right)^{1 / 2}}$.

This condition may be written as

$R_{0, \text { min }} \leq R_{0} \leq R_{0, \max }$,

which defines $R_{0, \max }$ and $R_{0, \min }$.

The assumed form of the magnetic field is used to represent horizontally localized, closed field line structures in the photosphere and chromosphere. It is shown in Sect. 4 that for a filling factor of 0.02 the resistive heating rate in these structures can balance the net radiative loss from the solar chromosphere if their characteristic diameters are sub-resolution, and if the magnetic field is highly twisted in that $B_{\theta} \gg B_{R}$. The form of sub-resolution magnetic structures in solar type stars may be very different from the one assumed here. However, it is essentially the high degree of horizontal localization of the magnetic field that gives rise to heating rates sufficiently large to balance the net radiative loss. The reason is that a higher degree of localization implies larger horizontal derivatives of the magnetic field components which implies larger current densities which implies larger heating rates for a given conductivity tensor. This suggests that magnetic fields that are highly localized in that their field strengths decrease by $\sim 1-2$ orders of magnitude from $\sim 10^{3} \mathrm{G}$ over horizontal distances $\lesssim 100 \mathrm{~km}$ have resistive heating rates that may be predicted with reasonable accuracy by any smoothly varying mathematical solution for such a localized field. It is proposed that if such localized magnetic fields exist in the photospheres and chromospheres of solar type stars, as they appear to in the solar photosphere, the model presented here may be used to predict their resistive heating rates with order of magnitude accuracy.

\subsection{Ohm's law}

Ohm's law is chosen to be

$$
\begin{aligned}
\boldsymbol{J} & =\bar{\sigma} \cdot\left(\boldsymbol{E}+\frac{\boldsymbol{V} \times \boldsymbol{B}}{c}\right) \\
& \equiv \bar{\sigma} \cdot \boldsymbol{E}^{\prime} .
\end{aligned}
$$

Here $\boldsymbol{E}, \boldsymbol{E}^{\prime}$, and $\bar{\sigma}$ are the electric field in the frame in which the $\mathrm{CM}$ velocity is $\boldsymbol{V}$, the $\mathrm{CM}$ electric field, and the total electrical conductivity tensor of the plasma. Once $\boldsymbol{J}$ is computed from $\boldsymbol{J}=(c / 4 \pi) \boldsymbol{\nabla} \times \boldsymbol{B}$, and the conductivity tensor is known from Sect. 2.5, Eq. (11) may be inverted to obtain the driving electric field $\boldsymbol{E}^{\prime}$. Then the heating rate per unit volume, discussed in more detail in Sect. 2.3 is given by $q=\boldsymbol{J} \cdot \boldsymbol{E}^{\prime}$.

Ohm's law may be written as

$\boldsymbol{J}=\sigma_{\|} \boldsymbol{E}_{\|}+\sigma_{\mathrm{P}} \boldsymbol{E}_{\perp}^{\prime}+\sigma_{\mathrm{H}} \boldsymbol{E}^{\prime} \times \hat{\boldsymbol{B}}$

where $\boldsymbol{E}_{\perp}^{\prime}$ is the CM electric field perpendicular to $\boldsymbol{B}, \boldsymbol{E}_{\|}$is the electric field parallel to $\boldsymbol{B}, \hat{\boldsymbol{B}}$ is a unit vector in the direction of $\boldsymbol{B}$, and $\sigma_{\|}, \sigma_{\mathrm{P}}$, and $\sigma_{\mathrm{H}}$ are the total parallel, Pedersen, and Hall conductivities. The corresponding components of $\boldsymbol{J}$ are the parallel, Pedersen, and Hall current densities.

\subsection{Resistive heating rates}

The resistive heating rate per unit volume is $q=\boldsymbol{J} \cdot \boldsymbol{E}^{\prime}$. Then from Eq. (12) it follows that

$$
\begin{aligned}
q & =\sigma_{\|} E_{\|}^{2}+\sigma_{\mathrm{P}}{E_{\perp}^{\prime}}^{2} \\
& \equiv q_{\|}+q_{\mathrm{P}} .
\end{aligned}
$$

$q_{\|}$and $q_{\mathrm{P}}$ are the heating rates due to dissipation of magnetic field aligned and Pedersen currents. The Hall current density is not dissipative since it is orthogonal to $\boldsymbol{E}^{\prime}$, and so cannot exchange energy with the electric field.

The Pedersen and Hall current densities are

$\boldsymbol{J}_{\mathrm{P}}=\sigma_{\mathrm{P}} \boldsymbol{E}_{\perp}^{\prime}$

$\boldsymbol{J}_{\mathrm{H}}=\sigma_{\mathrm{H}} \boldsymbol{E}_{\perp}^{\prime} \times \hat{\boldsymbol{B}}$.

Then

$J_{\perp}^{2}=J_{\mathrm{P}}^{2}+J_{\mathrm{H}}^{2}$

and $q_{\mathrm{P}}$ may be written as

$q_{\mathrm{P}}=\frac{\sigma_{\mathrm{P}} J_{\perp}^{2}}{\sigma_{\mathrm{P}}^{2}+\sigma_{\mathrm{H}}^{2}}$. 


\subsection{Plasma composition}

The plasma composition is the same as that assumed in Goodman (2004) where an averaging procedure is used to represent the solar atmosphere by a three species plasma. The procedure is outlined in this section. A detailed discussion of the procedure is given in Goodman (2004). The representation allows the conductivity tensor for a three species plasma of electrons, singly charged ions, and neutral particles derived by Mitchner \& Kruger (1978) to be used to describe resistive dissipation in the solar atmosphere.

The densities of electrons, protons, H, He I, He II, He III, C, $\mathrm{Si}, \mathrm{Al}, \mathrm{Mg}, \mathrm{Fe}, \mathrm{Na}$, and $\mathrm{Ca}$ are given by FAL CM.

The several species of singly charged metallic ions are collectively represented by a proxy ion, denoted by $i$, with a number density $n_{\mathrm{i}}$ and mass $m_{\mathrm{i}}$ defined by the quasi-neutrality condition

$n_{\mathrm{i}}=n_{\mathrm{e}}-\left(n_{\mathrm{p}}+n_{\mathrm{He} \text { II }}+2 n_{\mathrm{He} \text { III }}\right)$

and by

$m_{\mathrm{i}}=\frac{\rho_{\mathrm{C}}+\rho_{\mathrm{Si}}+\rho_{\mathrm{Al}}+\rho_{\mathrm{Mg}}+\rho_{\mathrm{Fe}}+\rho_{\mathrm{Na}}+\rho_{\mathrm{Ca}}}{n_{\mathrm{C}}+n_{\mathrm{Si}}+n_{\mathrm{Al}}+n_{\mathrm{Mg}}+n_{\mathrm{Fe}}+n_{\mathrm{Na}}+n_{\mathrm{Ca}}}$.

Here $n_{s}$ and $\rho_{s}=m_{s} n_{s}$ are the number and mass densities of particle species $s$.

The protons and proxy ions are collectively represented by an effective ion denoted by i, eff. Only its magnetization $M_{\mathrm{i}, \text { eff }}$ and mass $m_{\mathrm{i}, \text { eff }}$ enter the model. They are defined by

$$
\begin{aligned}
M_{\mathrm{i}, \mathrm{eff}} & =\frac{n_{\mathrm{i}} M_{\mathrm{i}}+n_{\mathrm{p}} M_{\mathrm{p}}}{n_{\mathrm{i}}+n_{\mathrm{p}}} \\
m_{\mathrm{i}, \mathrm{eff}} & =\frac{n_{\mathrm{i}} m_{\mathrm{i}}+n_{\mathrm{p}} m_{\mathrm{p}}}{n_{\mathrm{i}}+n_{\mathrm{p}}} .
\end{aligned}
$$

$M_{\mathrm{i}}$ and $M_{\mathrm{p}}$ are the magnetizations of proxy ions and protons. $\mathrm{He}$ II and He III are not included in the definition of the effective ion since they do not make a significant contribution to the Pedersen current in the photosphere or chromosphere due to their relatively small number densities.

$\mathrm{H}$ and $\mathrm{He} \mathrm{I}$ are collectively represented by a single neutral component with a mass

$$
m_{\mathrm{n}}=\frac{n_{\mathrm{H}} m_{\mathrm{p}}+n_{\mathrm{He}} m_{\mathrm{HeI}}}{n_{\mathrm{H}}+n_{\mathrm{He}}}
$$

The model plasma then consists of electrons, a singly charged ion species that collectively represents protons and metallic ions, and a neutral species that collectively represents $\mathrm{H}$ and $\mathrm{He} \mathrm{I}$.

\subsection{Conductivity tensors}

A three species total conductivity tensor derived by Mitchner \& Kruger (1978) is used for the model plasma defined in Sect. 2.4. This conductivity tensor is used by Goodman (2004) to derive the individual conductivity tensors for electrons and effective ions. The results are summarized in this section.

\subsubsection{Collision frequencies}

The magnetizations of electrons, protons, and proxy ions are defined by

$$
\begin{aligned}
& M_{\mathrm{e}}=\frac{\omega_{\mathrm{e}}}{v_{\mathrm{e}}^{*}} \\
& M_{\mathrm{p}}=\frac{\omega_{\mathrm{p}}}{v_{\mathrm{p}}^{*}} \\
& M_{\mathrm{i}}=\frac{\omega_{\mathrm{i}}}{v_{\mathrm{i}}^{*}} .
\end{aligned}
$$

The total reduced collision frequencies $v_{\mathrm{e}}^{*}, v_{\mathrm{p}}^{*}$, and $v_{\mathrm{i}}^{*}$ for electrons, protons, and proxy ions are defined by

$$
\begin{aligned}
& v_{\mathrm{e}}^{*}=v_{\mathrm{ep}}^{*}+v_{\mathrm{ei}}^{*}+v_{\mathrm{eH}}^{*}+v_{\mathrm{e}, \mathrm{He} I}^{*}+v_{\mathrm{e}, \mathrm{He} I I}^{*}+v_{\mathrm{e}, \mathrm{He} I I I}^{*} \\
& v_{\mathrm{p}}^{*}=v_{\mathrm{pe}}^{*}+v_{\mathrm{pi}}^{*}+v_{\mathrm{pH}}^{*}+v_{\mathrm{p}, \mathrm{He} \mathrm{I}}^{*}+v_{\mathrm{p}, \mathrm{He} I I}^{*}+v_{\mathrm{p}, \mathrm{He} I I I}^{*} \\
& v_{\mathrm{i}}^{*}=v_{\mathrm{ie}}^{*}+v_{\mathrm{ip}}^{*}+v_{\mathrm{iH}}^{*}+v_{\mathrm{i}, \mathrm{HeI}}^{*}+v_{\mathrm{i}, \mathrm{He} I \mathrm{II}}^{*}+v_{\mathrm{i}, \mathrm{He} I I I}^{*}
\end{aligned}
$$

Here $v_{k l}^{*}$ is the reduced collision frequency of a particle of species $k$ with particles of species $l$. It is defined by

$v_{k l}^{*}=v_{k l} \frac{m_{l}}{m_{k}+m_{l}}$,

where $v_{k l}$ is the collision frequency of a particle of species $k$ with particles of species $l$.

The collision frequencies $v_{k l}$ are given by standard expressions listed in Goodman (2004). They are functions of the temperature, and of the particle number densities and masses. The temperature and densities used here are from FAL CM. Since the FAL CM density and temperature profiles depend only on $z$, the model used here uses the approximation that these profiles are independent of $R$ although the magnetic field depends on $R$ and $z$. Then the conductivity tensors depend on $R$ only through the magnetic field.

\subsubsection{Conductivity tensors}

The elements of the total conductivity tensor are

$\sigma_{\|}=\sigma_{\|}^{(\mathrm{e})}$

$\sigma_{\mathrm{P}}=\frac{\sigma_{\|}(1+\Gamma)}{(1+\Gamma)^{2}+M_{\mathrm{e}}^{2}}$

$\sigma_{\mathrm{H}}=\frac{-M_{\mathrm{e}} \sigma_{\|}}{(1+\Gamma)^{2}+M_{\mathrm{e}}^{2}}$

$\sigma_{\|}^{(\mathrm{e})}=\frac{n_{\mathrm{e}} e^{2}}{m_{\mathrm{e}} v_{\mathrm{e}}^{*}}$

$\Gamma=\left(\frac{\rho_{\mathrm{n}}}{\rho}\right)^{2} M_{\mathrm{e}} M_{\mathrm{i}, \mathrm{eff}}$.

Here $\rho$ and $\rho_{\mathrm{n}}$ are the total and neutral mass densities defined by

$\rho=\rho_{\mathrm{e}}+\rho_{\mathrm{p}}+\rho_{\mathrm{i}}+\rho_{\mathrm{H}}+\rho_{\mathrm{He}}+\rho_{\mathrm{He} I \mathrm{I}}+\rho_{\mathrm{He} \mathrm{III}}$

$\rho_{\mathrm{n}}=\rho_{\mathrm{H}}+\rho_{\mathrm{He} \mathrm{I}}$,

and $\sigma_{\|}^{(\mathrm{e})}$ is the electron conductivity parallel to $\boldsymbol{B}$. 


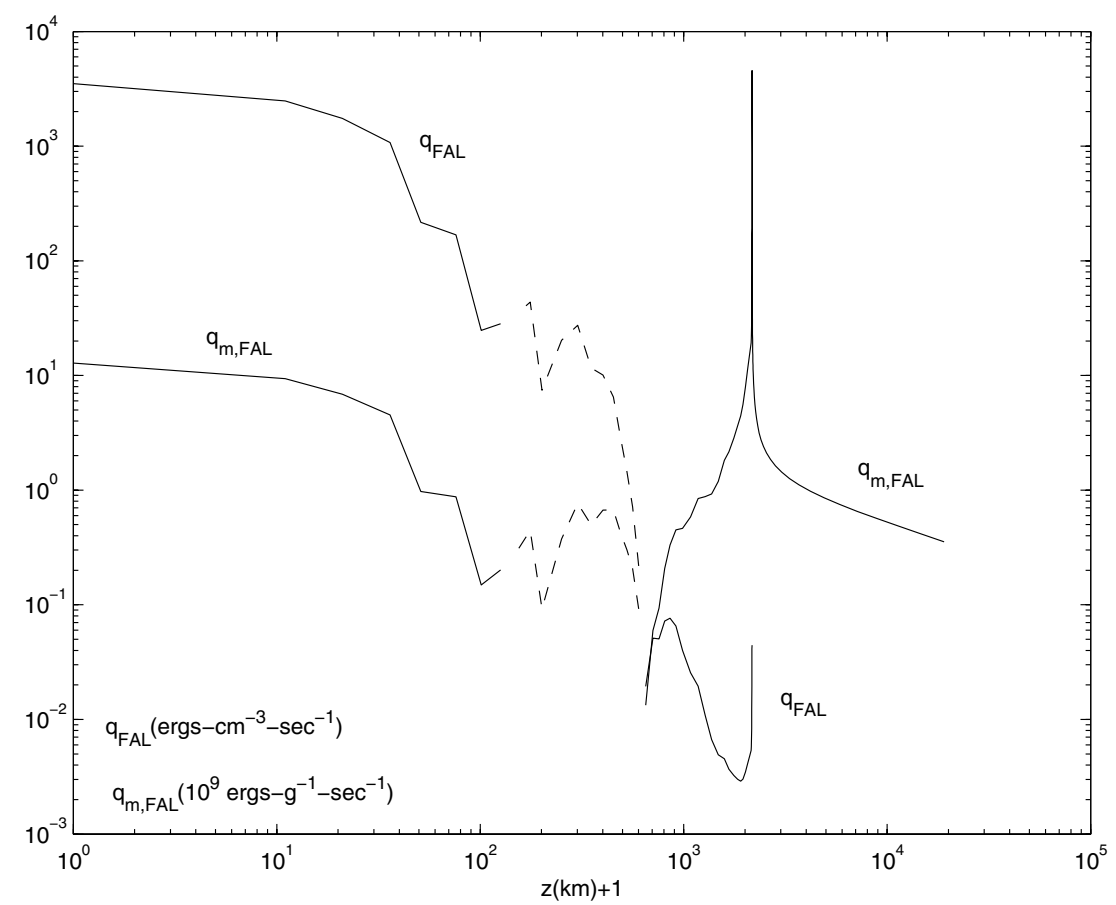

Fig. 1. FAL heating rates per unit volume, and per unit mass as a function of $z$. The dashed line denotes a negative heating rate. The units of the heating rate per unit mass are $10^{9} \mathrm{erg}^{-1} \mathrm{~s}^{-1}$.

The elements of the electron conductivity tensor are

$\sigma_{\|}^{(\mathrm{e})}=\sigma_{\|}$

$\sigma_{\mathrm{P}}^{(\mathrm{e})}=\frac{\sigma_{\|}}{(1+\Gamma)^{2}+M_{\mathrm{e}}^{2}}$

$\sigma_{\mathrm{H}}^{(\mathrm{e})}=\frac{-\sigma_{\|}\left(\Gamma(1+\Gamma)+M_{\mathrm{e}}^{2}\right)}{M_{\mathrm{e}}\left((1+\Gamma)^{2}+M_{\mathrm{e}}^{2}\right)}$.

The elements of the effective ion conductivity tensor are

$\sigma_{\|}^{(\mathrm{i}, \mathrm{eff})}=\mu \sigma_{\|}$

$\sigma_{\mathrm{P}}^{(\mathrm{i}, \mathrm{eff})}=(\Gamma+\mu) \sigma_{\mathrm{P}}^{(\mathrm{e})}$

$\sigma_{\mathrm{H}}^{(\mathrm{i}, \mathrm{eff})}=\left(\frac{\Gamma(1+\Gamma)}{M_{\mathrm{e}}}-\mu M_{\mathrm{e}}\right) \sigma_{\mathrm{P}}^{(\mathrm{e})}$

where

$\mu=\left(\frac{m_{\mathrm{e}}}{m_{(\mathrm{i}, \mathrm{eff}) \mathrm{n}}}\right)^{1 / 2} \frac{\rho_{\mathrm{n}}}{\rho} \ll 1$,

and

$m_{(\mathrm{i}, \mathrm{eff}) \mathrm{n}}=\frac{m_{(\mathrm{i}, \mathrm{eff})} m_{\mathrm{n}}}{m_{(\mathrm{i}, \mathrm{eff})}+m_{\mathrm{n}}}$

is the effective ion-neutral reduced mass.

In principle, the relations $\sigma_{\|}=\sigma_{\|}^{(\mathrm{e})}+\sigma_{\|}^{(\mathrm{i}, \text { eff })}, \sigma_{\mathrm{P}}=\sigma_{\mathrm{P}}^{(\mathrm{e})}+$ $\sigma_{\mathrm{P}}^{(\mathrm{i}, \text { eff })}$, and $\sigma_{\mathrm{H}}=\sigma_{\mathrm{H}}^{(\mathrm{e})}+\sigma_{\mathrm{H}}^{(\mathrm{i}, \text { eff })}$ should hold. Due to the approximate nature of the conductivity model these relations hold to within terms of order $\mu$.

\section{Solutions that represent magnetic structures for which the heating rate balances the chromospheric net radiative loss}

These solutions are obtained by choosing the model parameters to minimize the least squares difference between horizontal spatial averages of the model heating rate $q(R, z)$, and the total net radiative loss $q_{\text {FAL }}(z)$ computed by FAL CM and communicated by Avrett \& Loeser (2001). The procedure for doing this is as follows.

A solution to the model is determined by specifying the parameters $B_{0}, L_{B}, \tau$, and $R_{0}$ subject to the constraint Eq. (8). The temperature minimum of $4500 \mathrm{~K}$ occurs at $z=z_{T \text { min }}=525 \mathrm{~km}$. The height ranges of the photosphere and chromosphere are defined as $0 \leq z \leq z_{T \min }$ and $z_{T \min } \leq z \leq z_{\max }$.

$q_{\mathrm{FAL}}(z)$ is computed for $0 \leq z \leq 1.904 \times 10^{4} \mathrm{~km}$. It is shown in Fig. 1. The dashed section of the curve extends from $z=150 \mathrm{~km}$ to $z=600 \mathrm{~km}$, and indicates where $q_{\mathrm{FAL}}<0$. The first height above $600 \mathrm{~km}$ where FAL CM predicts a positive heating rate is $650 \mathrm{~km}$.

For a given radius $R^{*}$, define the horizontal average of $q(R, z)$ over an area $A=\pi R_{*}^{2}$ of the magnetic structure by

$\langle q\rangle_{R^{*}}(z)=\frac{2 \pi}{A} \int_{0}^{R^{*}} q(R, z) R \mathrm{~d} R$.

Similarly, noting that $q(R, z)=q_{\|}(R, z)+q_{\mathrm{P}}(R, z)$, define

$\left\langle q_{\|}\right\rangle_{R^{*}}(z)=\frac{2 \pi}{A} \int_{0}^{R_{*}} q_{\|}(R, z) R \mathrm{~d} R$

$\left\langle q_{\mathrm{P}}\right\rangle_{R^{*}}(z)=\frac{2 \pi}{A} \int_{0}^{R^{*}} q_{\mathrm{P}}(R, z) R \mathrm{~d} R$.

$R^{*}$ must be chosen large enough so that $A$ includes the most strongly heated plasma, but not so large that the average 


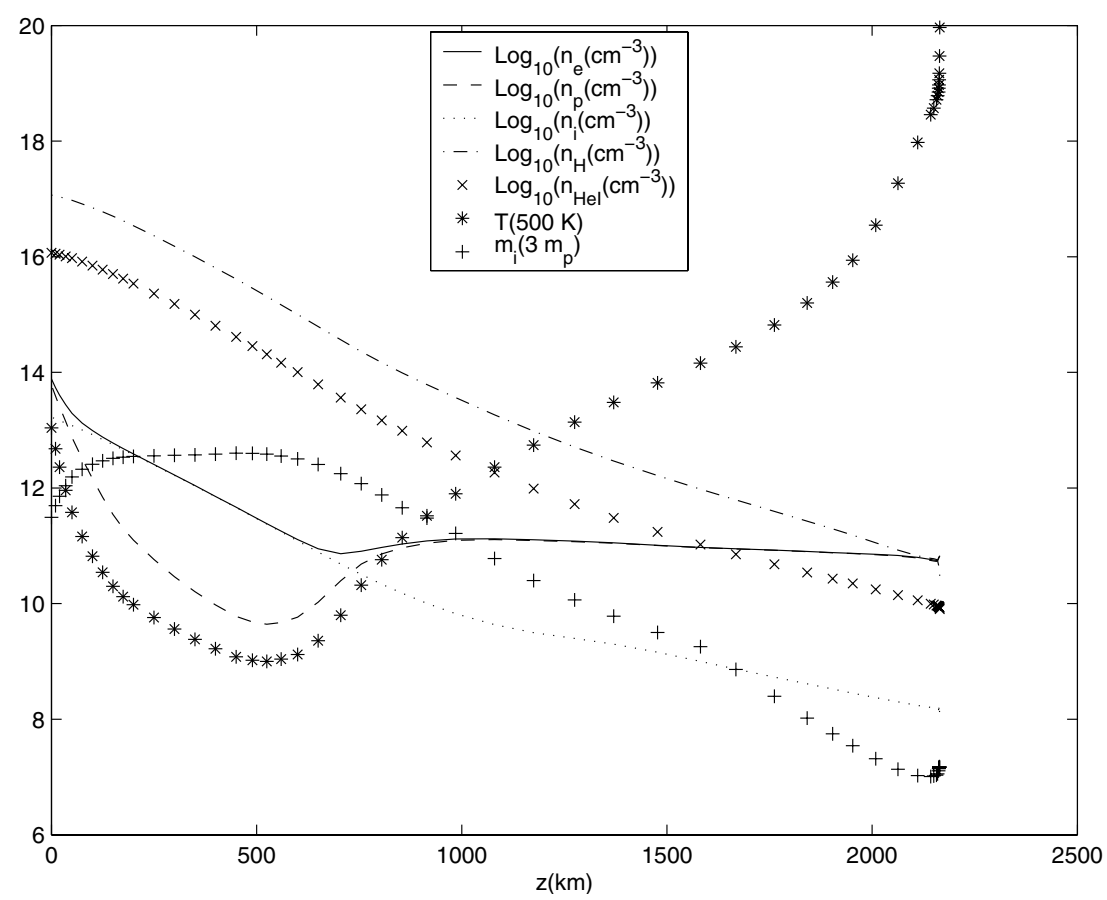

Fig. 2. Electron, proton, proxy ion, $\mathrm{H}$, and He I number densities, temperature, and proxy ion mass as functions of $z$. The temperature is given in units of $500 \mathrm{~K}$. The proxy ion mass is given in units of three times the proton mass.

heating rate is greatly reduced by including weakly heated plasma. $q(R, z)$ is found to decrease (increase) rapidly with decreasing (increasing) $B_{0}$. The choices $R^{*}=R_{0}, 2 R_{0}$ are made, and for each choice of $R^{*}$ solutions corresponding to $R_{0}=R_{0, \text { min }}$ and $R_{0}=R_{0, \text { max }}$ are generated. These choices are made since it is found that the resistive heating is strongly concentrated in the core region.

For each of the four choices for $R^{*}$, some or all of the parameters $B_{0}, L_{B}, \tau$, and the filling factor $f$ are to be varied to minimize the least squares difference

$\chi^{2}=\int_{650}^{z_{\max }}\left(f\langle q\rangle_{R^{*}}(z)-q_{\mathrm{FAL}}(z)\right)^{2} \mathrm{~d} z$,

where $z$ is in kilometers. The lower limit of integration is chosen as the minimum height above the height of the temperature minimum at which $q_{\text {FAL }}>0$. Some of the parameters may be fixed by observations or ansatz. The remaining ones are to be varied. A particular case of this minimization procedure is used in Sect. 4.1 to show that Pedersen current dissipation in magnetic structures with sub-resolution horizontal dimensions, kilogauss photospheric field strengths, and $f \sim 0.02$ can globally balance the chromospheric net radiative loss. For this case, $B_{0}$ and $f$ are taken from observation, $L_{B}$ is fixed at $600 \mathrm{~km}$, and $\tau$ is varied to minimize $\chi^{2}$.

\section{Heating in small scale magnetic structures}

Domínguez Cerdeña et al. (2003) detect a kilogauss strength magnetic field structure in the photospheric internetwork that is inferred to have $D<180 \mathrm{~km}$, and to belong to a class of similar structures having $f \sim 2 \%$. Supporting observations of Sánchez Almeida et al. (2003) (also see Socas-Navarro \& Sánchez Almeida 2003) indicate that most of the unsigned magnetic flux in the photospheric internetwork is in the form of kilogauss strength magnetic field concentrations, and that fields with strengths $\sim 1100 \mathrm{G}$ and $\sim 300 \mathrm{G}$ have $f \sim 1 \%$ and $f \sim 2 \%$.

It is shown in this section that if $B_{0}$ and $f$ are set equal to $1000 \mathrm{G}$ and 0.02 as suggested by observation, and if $L_{B}$ is chosen to be $600 \mathrm{~km}$, then the values of $\tau$ that minimize $\chi^{2}$ yield values for the characteristic diameter $D \equiv 2 R^{*}$ for the four choices of $R^{*}$ defined after Eq. (48) that are in the range of $98-161 \mathrm{~km}$. This range of $D$ is consistent with the observational estimate that $D<180 \mathrm{~km}$.

$L_{B}$ is fixed at the value of $600 \mathrm{~km}$ since this implies that if $B(0,0)=1000 \mathrm{G}$, then $B\left(0, z_{\max }\right)=27 \mathrm{G}$, which is assumed to be a reasonable field strength in the upper chromosphere. Larger (smaller) values of $L_{B}$ lead to larger (smaller) heating rates.

\subsection{Numerical results I}

The four choices for $R^{*}$ are $R_{0, \max }, R_{0, \min }, 2 R_{0, \max }$, and $2 R_{0, \min }$. The solution corresponding to $R^{*}=2 R_{0, \max }$ is presented in detail in this section. The other three solutions have similar properties, and are briefly discussed in Sect. 4.2.

Let $R^{*}=2 R_{0}=2 R_{0, \max }$. Then the value of $\tau$ that minimizes $\chi^{2}$ is 59.5 , and $D=161.32 \mathrm{~km}$. The magnetic structure is highly twisted in that $B_{\theta} \gg B_{R}$, which is the case for the other three solutions discussed in Sect. 4.2.

Figure 1 shows the FAL CM heating rates per unit volume, and per unit mass from the photosphere to the lower corona.

Figure 2 shows the FAL CM profiles of the electron, proton, $\mathrm{H}, \mathrm{He} \mathrm{I}$, and proxy ion number densities, ion mass, and temperature that are used as inputs to the model. 


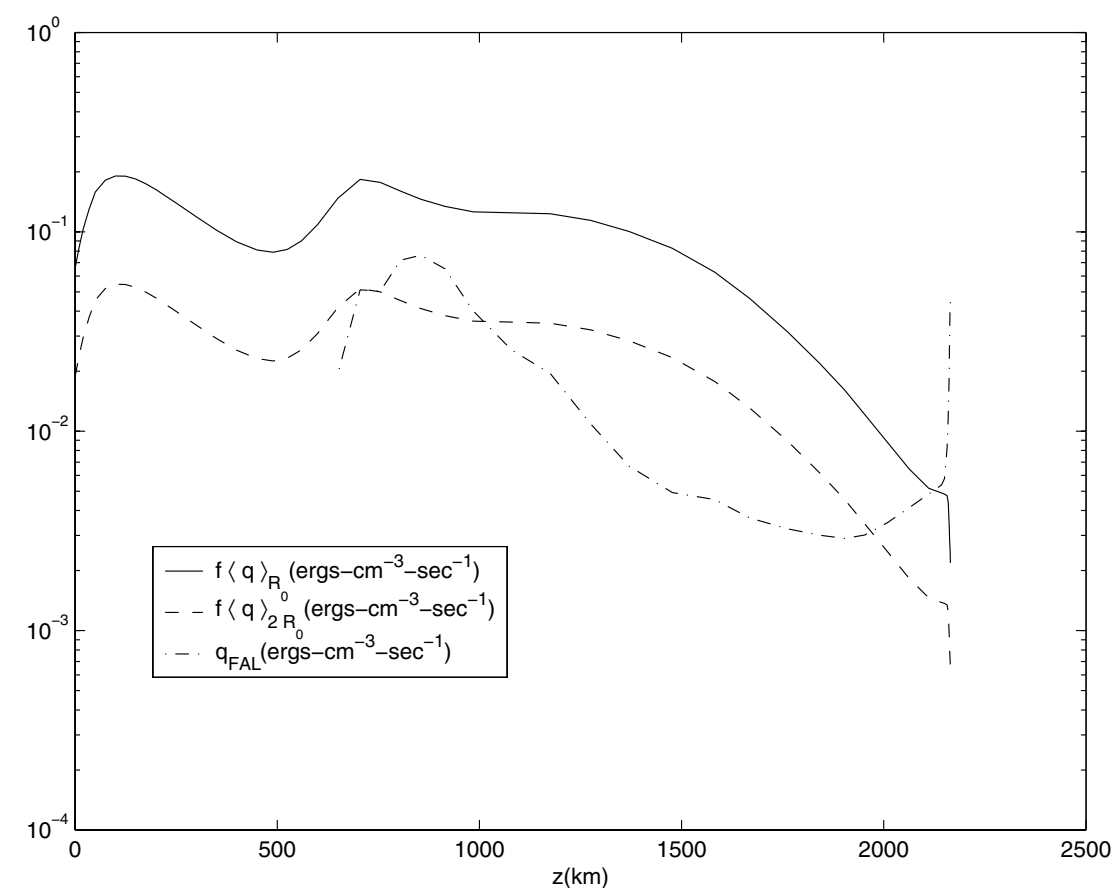

Fig. 3. Area averaged heating rates per unit volume, weighted by filling factor, and the FAL heating rate per unit volume as functions of $z$.

Figure 3 shows the result of minimizing the difference between $q_{\mathrm{FAL}}(z)$ and $f\langle q\rangle_{2 R_{0}}$ over the height range of $650 \leq z \leq z_{\max } \cdot f\langle q\rangle_{2 R_{0}}(z) / q_{\mathrm{FAL}}(z) \lesssim 2.8$ for $650 \leq z \lesssim$ $2063 \mathrm{~km}$, and is $\lesssim 10$ for $2063 \lesssim z \lesssim 2163 \mathrm{~km}$. The larger differences at greater heights indicate that the model rapidly breaks down with increasing height near the base of the transition region. It is shown in Goodman (2004) that Pedersen current dissipation is not important in the transition region and lower corona on the vertical spatial scales of the one dimensional FAL CM model. Its importance on smaller spatial scales remains to be determined. The photospheric heating rate for the model solution is several orders of magnitude smaller than the FAL CM photospheric heating rate in Fig. 1. Goodman (2004) shows that Pedersen current dissipation is not important below $z_{T \text { min }}$ since in that region $\sigma_{\mathrm{P}} \sim \sigma_{\|}$, so there is no qualitative difference between the mechanisms of Pedersen and parallel current dissipation. There $q \sim \sigma_{\|} E^{\prime 2}$. Figure 3 also shows the core heating rate $f\langle q\rangle_{R_{0}}(z)$.

Define the horizontally averaged chromospheric heating flux by

$F_{R^{*}}=f \int_{z_{\text {min }}}^{z_{\max }}\langle q\rangle_{R^{*}}(z) \mathrm{d} z$

For the profiles in Fig. $2, F_{R_{0}}=1.5 \times 10^{7} \mathrm{erg} \mathrm{cm}^{-2} \mathrm{~s}^{-1}$, and $F_{2 R_{0}}=4.2 \times 10^{6} \mathrm{erg} \mathrm{cm}^{-2} \mathrm{~s}^{-1}$. These values are close to the observed chromospheric net radiative loss of $\sim 10^{7} \mathrm{erg} \mathrm{cm}^{-2} \mathrm{~s}^{-1}$. The fraction of $F_{R_{0}}$ and $F_{2 R_{0}}$ that is due to Pedersen current dissipation is $\sim 0.95$, the remainder being due to parallel current dissipation strongly concentrated near the temperature minimum.

Figure 4 shows the result of dividing the profiles in Fig. 3 by $\rho(z)$ to obtain the model chromospheric heating rates per unit mass of $q_{m, \mathrm{FAL}}(z), f\left\langle q_{m}\right\rangle_{R_{0}}$, and $f\left\langle q_{m}\right\rangle_{2 R_{0}}(z) . f\left\langle q_{m}\right\rangle_{R_{0}}(z)$ and $f\left\langle q_{m}\right\rangle_{2 R_{0}}(z)$ reach $10^{9} \mathrm{erg} \mathrm{g}^{-1} \mathrm{~s}^{-1}$ at $z \sim 925 \mathrm{~km}$ and $z \sim 1100 \mathrm{~km}$. They reach maximum values of $\sim 2.8 \times$ $10^{10} \mathrm{erg} \mathrm{g}^{-1} \mathrm{~s}^{-1}$ and $\sim 7.8 \times 10^{9} \mathrm{erg} \mathrm{g}^{-1} \mathrm{~s}^{-1}$. Their range of values from $z \sim 1000 \mathrm{~km}$ up to the transition region is close to the constant value of $4.5 \times 10^{9} \mathrm{erg} \mathrm{g}^{-1} \mathrm{~s}^{-1}$ that the one dimensional, semi-empirical model of Anderson \& Athay (1989a,b) predicts for the height range of $1000-2000 \mathrm{~km}$.

Define the volume averaged heating rate per unit mass by

$F_{m, R^{*}}=\frac{f}{z_{\max }-z_{T \min }} \int_{z_{T \min }}^{z_{\max }}\left\langle q_{m}\right\rangle_{R^{*}}(z) \mathrm{d} z$.

For the profiles in Fig. $4, F_{m, R_{0}}=1.2 \times 10^{10} \mathrm{erg} \mathrm{g}^{-1} \mathrm{~s}^{-1}$, and $F_{m, 2 R_{0}}=3.4 \times 10^{9} \mathrm{erg} \mathrm{g}^{-1} \mathrm{~s}^{-1}$. The corresponding value computed by averaging $q_{m, \mathrm{FAL}}(z)$ over the same height range is $2.6 \times 10^{9} \mathrm{erg} \mathrm{g}^{-1} \mathrm{~s}^{-1}$. These values are close to one another, and to the constant Anderson \& Athay (1989a,b) value. The fractions of $F_{m, R_{0}}$ and $F_{m, 2 R_{0}}$ that are due to Pedersen current dissipation are $\sim 0.999$, the remainder being due to parallel current dissipation.

The conditions under which Pedersen current dissipation can be important as a heating mechanism from the photosphere to the lower corona are determined by Goodman (2004). Some of the main conclusions of Goodman (2004) are illustrated in Figs. 5-7. Together with Figs. 8-10 they show that the region of the atmosphere in which Pedersen current dissipation is highly efficient and qualitatively different from parallel current dissipation is also the region where the associated heating rates per unit volume and mass are largest. This region is the chromosphere, and these heating rates are one or more orders of magnitude larger in the chromosphere than in the photosphere.

Figure 5 shows the horizontally averaged magnetizations of electrons, protons, proxy ions, and effective ions as a function of height. $\left\langle M_{s}\right\rangle_{R_{0}}$ and $\left\langle M_{s}\right\rangle_{R_{0}-2 R_{0}}$ are averages of $M_{s}(R, z)$ over the areas defined by $0 \leq R \leq R_{0}$ and $R_{0} \leq R \leq 2 R_{0}$. The averages of $M_{\mathrm{i}, \text { eff }}$ and $M_{\mathrm{p}}$ are essentially equal. When $M_{s}$ 


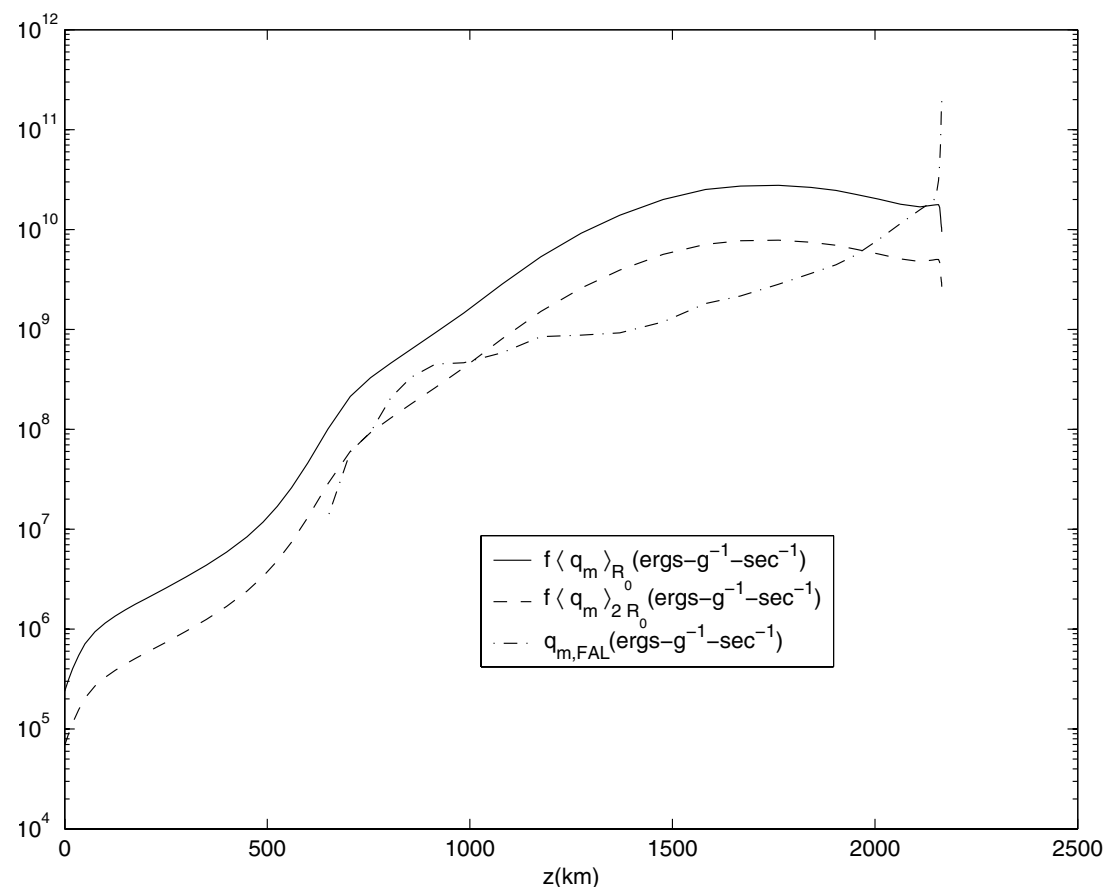

Fig. 4. Area averaged heating rates per unit mass, weighted by filling factor, and the FAL heating rate per unit mass as functions of $z$.

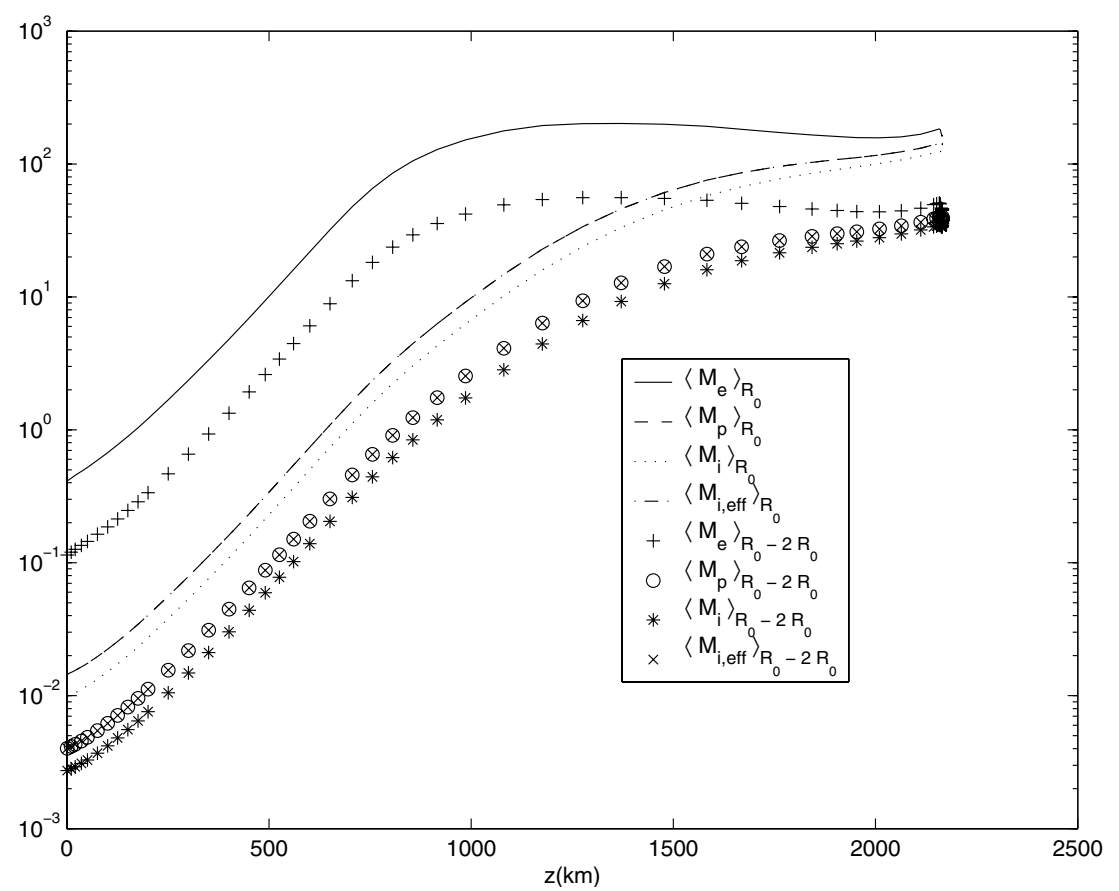

Fig. 5. Electron, proton, proxy ion, and effective ion magnetizations averaged over the areas defined by $0 \leq R \leq R_{0}$ (denoted by $R_{0}$ ) and $R_{0} \leq R \leq 2 R_{0}$ (denoted by $R_{0}-2 R_{0}$ ) as functions of $z$.

increases through unity, species $s$ is said to become magnetized. $\left\langle M_{s}\right\rangle_{R_{0}}=1$ for electrons, protons, and proxy ions at $z \sim 175,650$, and $700 \mathrm{~km} .\left\langle M_{s}\right\rangle_{R_{0}-2 R_{0}}=1$ for electrons, protons, and proxy ions at $z \sim 355,822$, and $885 \mathrm{~km}$.

Figure 6 shows the efficiency $Q(R, z)$ of Pedersen current dissipation, discussed in detail in Goodman (2004). By definition, $Q=q_{\mathrm{P}} / q_{\mathrm{P}, \max }$, where $q_{\mathrm{P}, \max }=J_{\perp}^{2} / \sigma_{\mathrm{P}}$ is the maximum possible value of $q_{\mathrm{P}}$ for a given value of $J_{\perp}$. $Q=1 \Leftrightarrow \boldsymbol{J}_{\perp} \| \boldsymbol{E}_{\perp}^{\prime} . Q$ is the efficiency with which the driving electric field $\boldsymbol{E}_{\perp}^{\prime}$ transfers energy to the current density that is dissipated by collisions. From Goodman (2004), $Q=\left(1+\left(\sigma_{\mathrm{H}} / \sigma_{\mathrm{P}}\right)^{2}\right)^{-1}$. Then Pedersen current dissipation is efficient in that $Q \sim 1 \Leftrightarrow \sigma_{\mathrm{P}} \gg \sigma_{\mathrm{H}}$. Figure 7 shows $\sigma_{\|}(z) / \sigma_{\mathrm{P}}(R, z)$. Pedersen current dissipation is a qualitatively different heating mechanism from parallel current dissipation $\Leftrightarrow \sigma_{\mathrm{P}} \ll \sigma_{\|}$. Then Pedersen current dissipation is efficient and qualitatively different from parallel current dissipation, and hence can be a distinct and important heating 


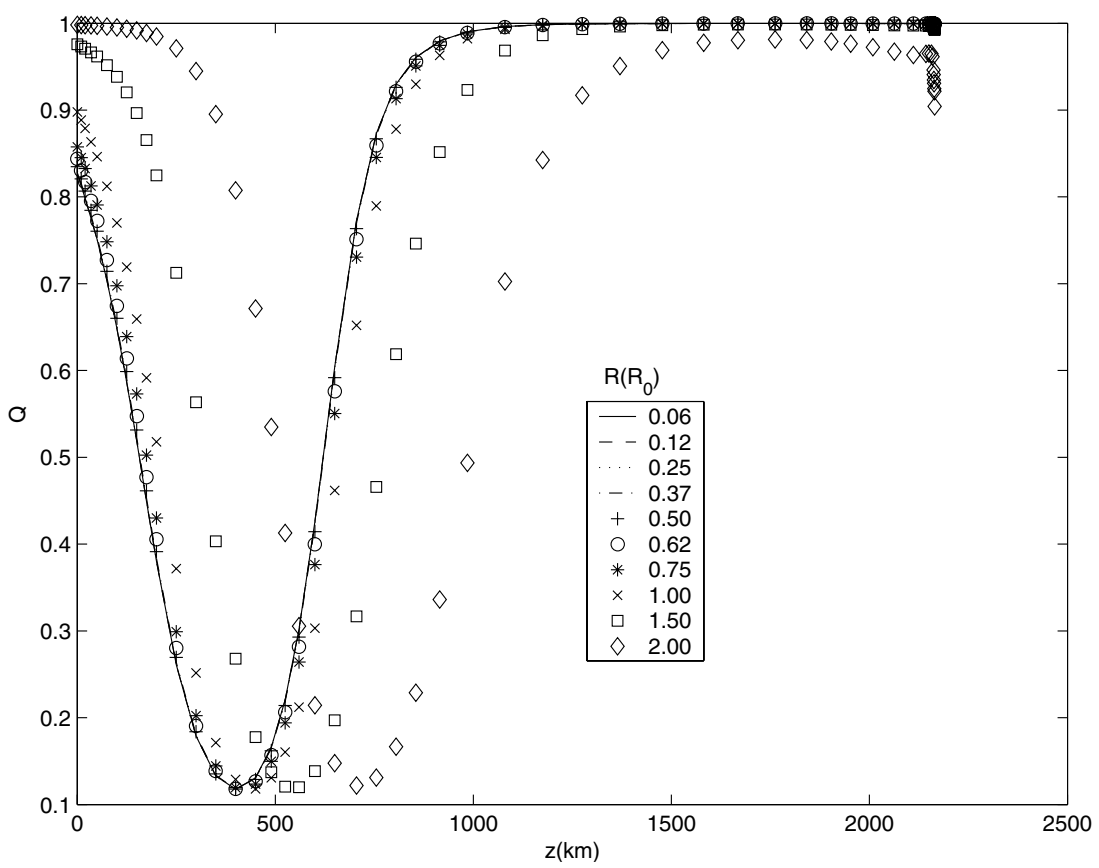

Fig. 6. Efficiency of Pedersen current dissipation as a function of $R$ and $z$.

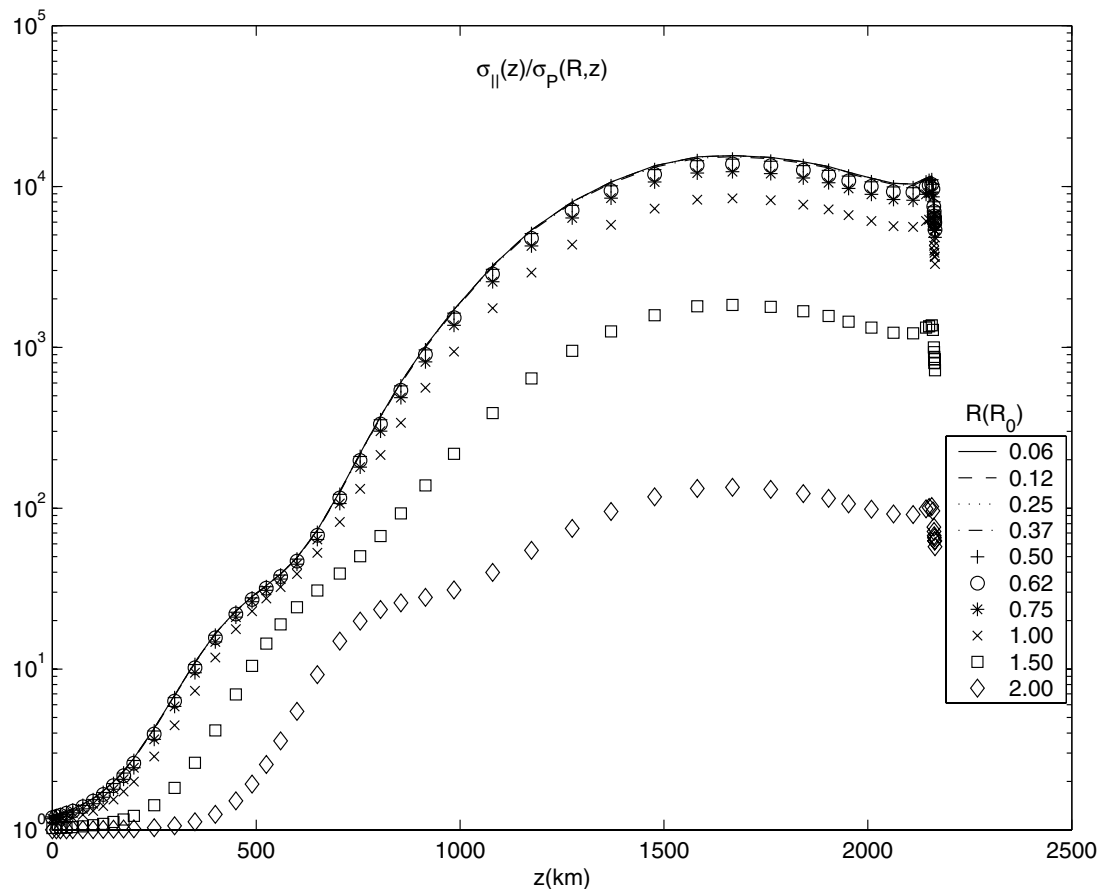

Fig. 7. Ratio of parallel to Pedersen conductivity as a function of $R$ and $z$.

mechanism $\Leftrightarrow \sigma_{\mathrm{H}} \ll \sigma_{\mathrm{P}} \ll \sigma_{\|}$. Figures 5-7 show that this condition does not hold in the photosphere but does hold in the chromosphere, and that there is a transition beginning near the temperature minimum between the photosphere where the condition is not satisfied, and the chromosphere where it is satisfied. This transition is caused by the magnetization of the ions and protons that occurs near the temperature minimum. This region of the atmosphere is weakly ionized in that collisional momentum transfer to the charged particles is due almost entirely to collisions with $\mathrm{H}$. Then $M_{s} \propto B T^{1 / 2} / n_{\mathrm{H}}$. This shows that, since $T^{1 / 2}$ varies slowly, it is the steady decrease of $n_{\mathrm{H}}$ relative to $B$ with height that causes the charged particles to become magnetized.

Figure 8 shows $q_{\mathrm{P}}(R, z)$. In the core region, where almost all of the heating occurs, $q_{\mathrm{P}}$ increases with height by $\sim$ one order of magnitude from a local minimum at $z \sim 400 \mathrm{~km}$ to its global maximum at $z \sim 700 \mathrm{~km}$, and then decreases relatively slowly with increasing height. The height range $\Delta z=400-700 \mathrm{~km}$ is centered at the height of the temperature minimum. There 


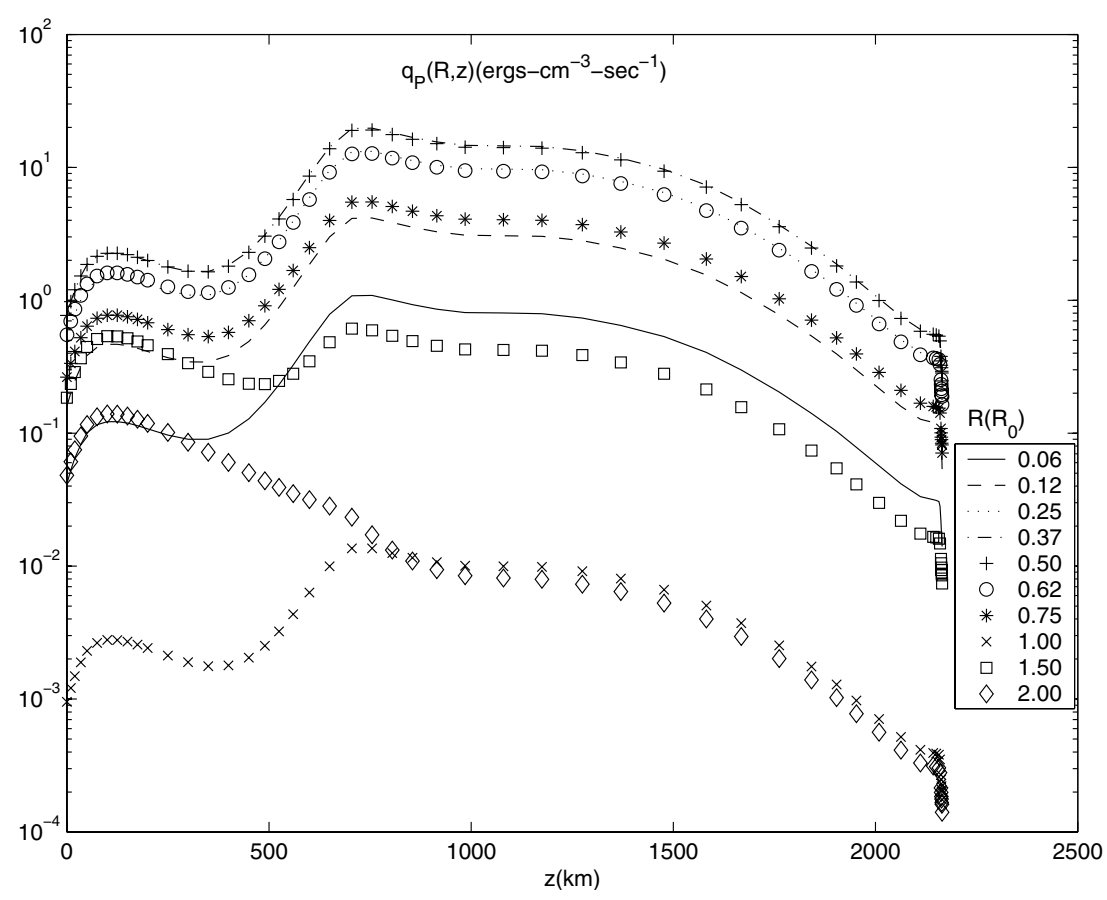

Fig. 8. Heating rate per unit volume as a function of $R$ and $z$.

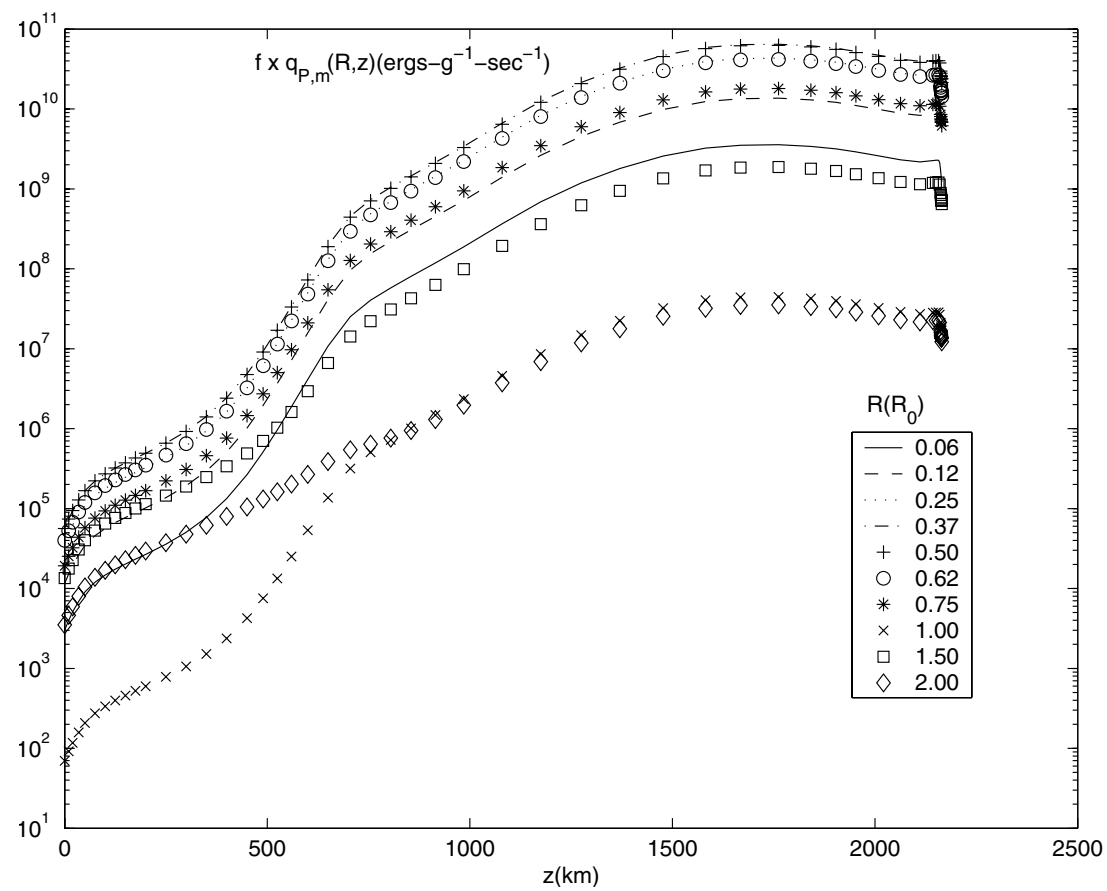

Fig. 9. Heating rate per unit mass as a function of $R$ and $z$, multiplied by the filling factor $f=0.02$.

is a direct correlation between the chromospheric temperature inversion and the initial rapid increase in $q_{\mathrm{P}}$ with height in the sub-resolution core region of the magnetic structure.

Figures 6 and 7 show that the inequalities $\sigma_{\mathrm{H}} \ll \sigma_{\mathrm{P}} \ll \sigma_{\|}$ first become simultaneously satisfied in $\Delta z$. Goodman (2004) shows that these inequalities are simultaneously satisfied $\Leftrightarrow M_{\mathrm{e}} \gg 1$ and $M_{\mathrm{i}, \text { eff }} \gtrsim 2$. In the core region, $M_{\mathrm{e}}$ and $M_{\mathrm{i}, \text { eff }}\left(\sim M_{\mathrm{p}}\right)$ increase from $\sim 4-5$ and $\sim 0.1-0.2$ to $\sim 40-50$ and $\sim 1-2$ over $\Delta z$. In this region, $M_{\mathrm{p}}=1$ at $z \sim 650 \mathrm{~km}$, where $q_{\mathrm{FAL}}$ first becomes positive above $z_{T \text { min }}$. From Goodman (2004), $\sigma_{\|} / \sigma_{\mathrm{P}} \sim 1+M_{\mathrm{e}} M_{\mathrm{i}, \text { eff }}+M_{\mathrm{e}}^{2} /\left(1+M_{\mathrm{e}} M_{\mathrm{i}, \mathrm{eff}}\right)$ since $\rho_{\mathrm{n}} \sim \rho$ in $\Delta z$. Then $\sigma_{\|} / \sigma_{\mathrm{P}} \sim 13-126$ in $\Delta z$, so Pedersen current dissipation is significantly different from parallel current dissipation throughout $\Delta z$. Again from Goodman (2004), $\sigma_{\mathrm{P}} / \sigma_{\mathrm{H}} \sim M_{\mathrm{i}, \mathrm{eff}}+1 / M_{\mathrm{e}}$, which in the core region ranges from $\sim 0.35$ at $z=400 \mathrm{~km}$ to $\sim 2$ at $z=700 \mathrm{~km}$, corresponding to an increase of $Q$ from its minimum of $\sim 0.10$ to $\sim 0.80$. As the protons become magnetized in $\Delta z$, Pedersen current dissipation becomes an efficient channel for dissipating the energy in $\boldsymbol{J}_{\perp}$. This process is directly correlated with the increase of $q_{\mathrm{P}}$ with height by an order of 


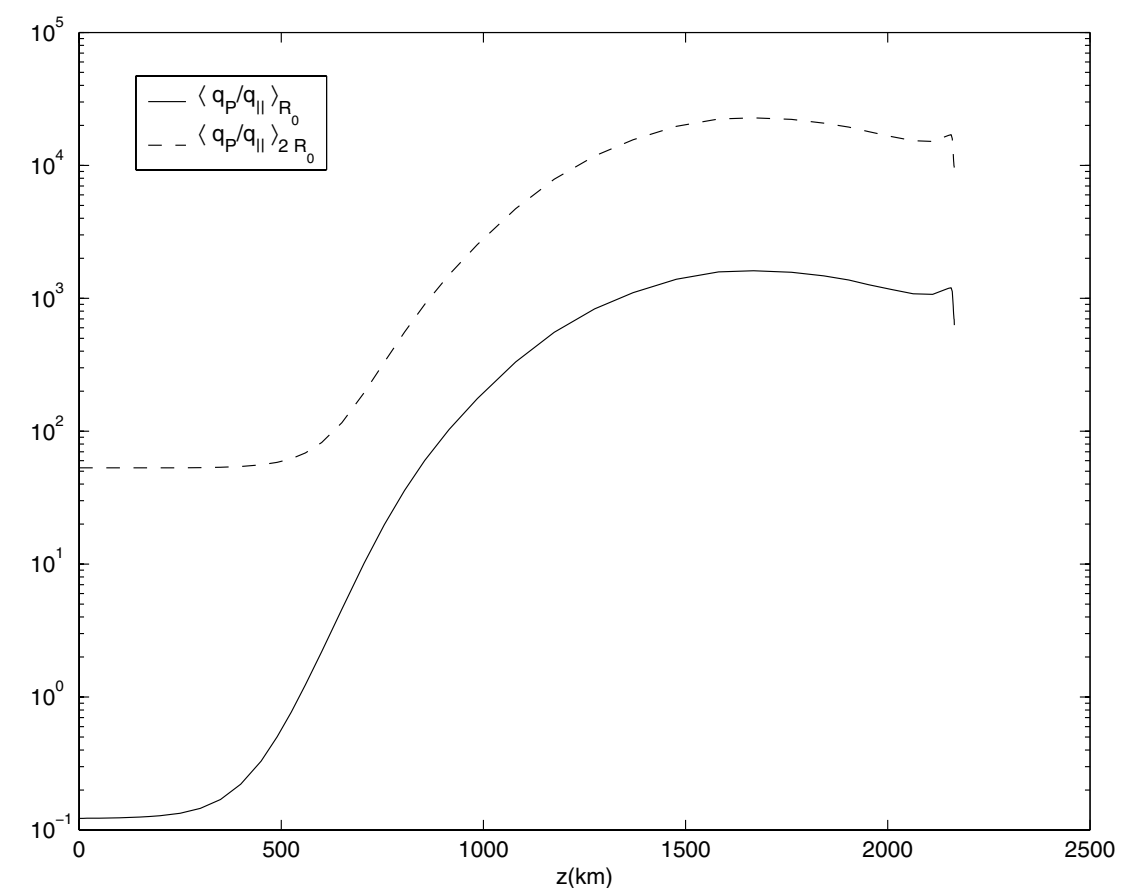

Fig. 10. Ratio of Pedersen current dissipation rate to parallel current dissipation rate, averaged over areas with radii of $R_{0}$ and $2 R_{0}$, as functions of $z$.

magnitude in $\Delta z$, and with the chromospheric temperature inversion.

Figure 9 shows $f q_{\mathrm{P}, \mathrm{m}}(R, z)$. Its values in the core region are $\sim 10^{9}-10^{11} \mathrm{erg} \mathrm{g}^{-1} \mathrm{~s}^{-1}$ for $z \gtrsim 10^{3} \mathrm{~km}$. Horizontal averaging of these values occurs during observations with a spatial resolution greater than the core diameter $D_{0}=2 R_{0}$. For the four cases considered here $D_{0} \sim 49-152 \mathrm{~km} \sim 0.07^{\prime \prime}-0.21^{\prime \prime}$. This range of $D_{0}$ is currently sub-resolution for detecting magnetic structures. At a resolution much larger than $D_{0}$, heating in an approximately uniform distribution of similar magnetic structures with filling factor $f$ appears as a heating rate per unit mass that varies weakly with horizontal position, and has a height dependence similar to that predicted by Anderson \& Athay (1989a,b) above the height of the temperature minimum. The temperature minimum results from the horizontal average over the heights of the temperature minima of the individual structures.

Figure 10 shows $\left\langle q_{\mathrm{P}} / q_{\|}\right\rangle_{R^{*}}(z)$ for $R^{*}=R_{0}$, and $2 R_{0}$. In the core region, parallel current dissipation dominates in the photosphere, below $z_{T \text { min }}$. Beginning near $z_{T \text { min }}$ Pedersen current dissipation rapidly dominates parallel current dissipation in the core region, exceeding it by orders of magnitude for $z \gtrsim 700 \mathrm{~km}$. For $R^{*}=2 R_{0}$, Pedersen current dissipation exceeds parallel current dissipation everywhere by one or more orders of magnitude.

Figures 11 and 12 show the magnitudes of the components $\boldsymbol{E}_{\perp}^{\prime}$ and $\boldsymbol{E}_{\|}$of the driving electric field $\boldsymbol{E}^{\prime}$. These components drive the Pedersen and parallel currents. $E_{\|}>E_{\perp}^{\prime}$ in some regions of the photosphere below $z_{T \text { min }}$. Beginning near $z_{T \text { min }}$, $E_{\perp}^{\prime}$ rapidly increases, and exceeds $E_{\|}$by orders of magnitude for $z \gtrsim z_{T \text { min }}$.

Figures 13-15 show the total parallel, Pedersen, and Hall conductivities. They vary by orders of magnitude, and can differ by orders of magnitude throughout the magnetic structure.
This behavior has a strong effect in determining the resistive heating rate, and underscores the importance of using realistic representations of transport processes in models of the solar atmosphere. The behavior of $\sigma_{\mathrm{P}}$ in Fig. 14, and $E_{\perp}^{\prime}$ in Fig. 11 are inversely correlated. This suggests that the decrease of $\sigma_{\mathrm{P}}$ with increasing height allows $E_{\perp}^{\prime}$ to build up to sufficiently large values to cause $q_{\mathrm{P}}\left(\equiv \sigma_{\mathrm{P}}{E^{\prime}}^{2}\right)$ to become significant, meaning comparable to the chromospheric net radiative loss. The particle magnetizations are so small in the photosphere that $\sigma_{\mathrm{P}}$ is close to its maximum possible value of $\sigma_{\|}$. These relatively large values of $\sigma_{\mathrm{P}}$ prevent $E_{\perp}^{\prime}$, and hence $q_{\mathrm{P}}$ from being significant. There is a similar correlation between the behavior of $\sigma_{\|}$in Fig. 13, and $E_{\|}$in Fig. 12, suggesting that the increase of $\sigma_{\|}$with height prevents $E_{\|}$from building up to sufficiently large values to cause $q_{\|}\left(\equiv \sigma_{\|} E_{\|}^{2}\right)$ to be significant in the chromosphere.

Figure 16 shows the relative contribution $J_{\mathrm{P}}^{(\mathrm{i}, \text { eff })} / J_{\mathrm{P}}^{(\mathrm{e})}$ of effective ions and electrons to the Pedersen current. The effective ions dominate the Pedersen current for $z \gtrsim 450 \mathrm{~km}$. The effective ions are a composite of protons and proxy ions. Figure 17 shows the relative contribution $J_{\mathrm{P}}^{(\mathrm{p})} / J_{\mathrm{P}}^{(\mathrm{i})}$ of protons and proxy ions to the effective ion Pedersen current. Except in the height range of $z \sim 180-580 \mathrm{~km}, J_{\mathrm{P}}^{(\mathrm{p})}>J_{\mathrm{P}}^{(\mathrm{i})} . J_{\mathrm{P}}^{(\mathrm{p})} \sim 5 J_{\mathrm{P}}^{(\mathrm{i})}$ at $z \sim 680 \mathrm{~km}$, and $J_{\mathrm{P}}^{(\mathrm{p})} \sim 10 J_{\mathrm{P}}^{(\mathrm{i})}$ at $z \sim 700 \mathrm{~km}$. Since, as discussed in the context of Fig. 8, $q_{\mathrm{P}}$ increases rapidly from a local minimum to its global maximum over the height range 400-700 km, the dissipation of metallic ion Pedersen current makes an important contribution to chromospheric heating up to $z \sim 680 \mathrm{~km}$. Goodman (2000) proposes that it is initially metallic ion Pedersen current dissipation below the height of the temperature minimum in a magnetic structure that starts the heating process leading to the chromospheric temperature inversion that is then maintained by proton Pedersen current 


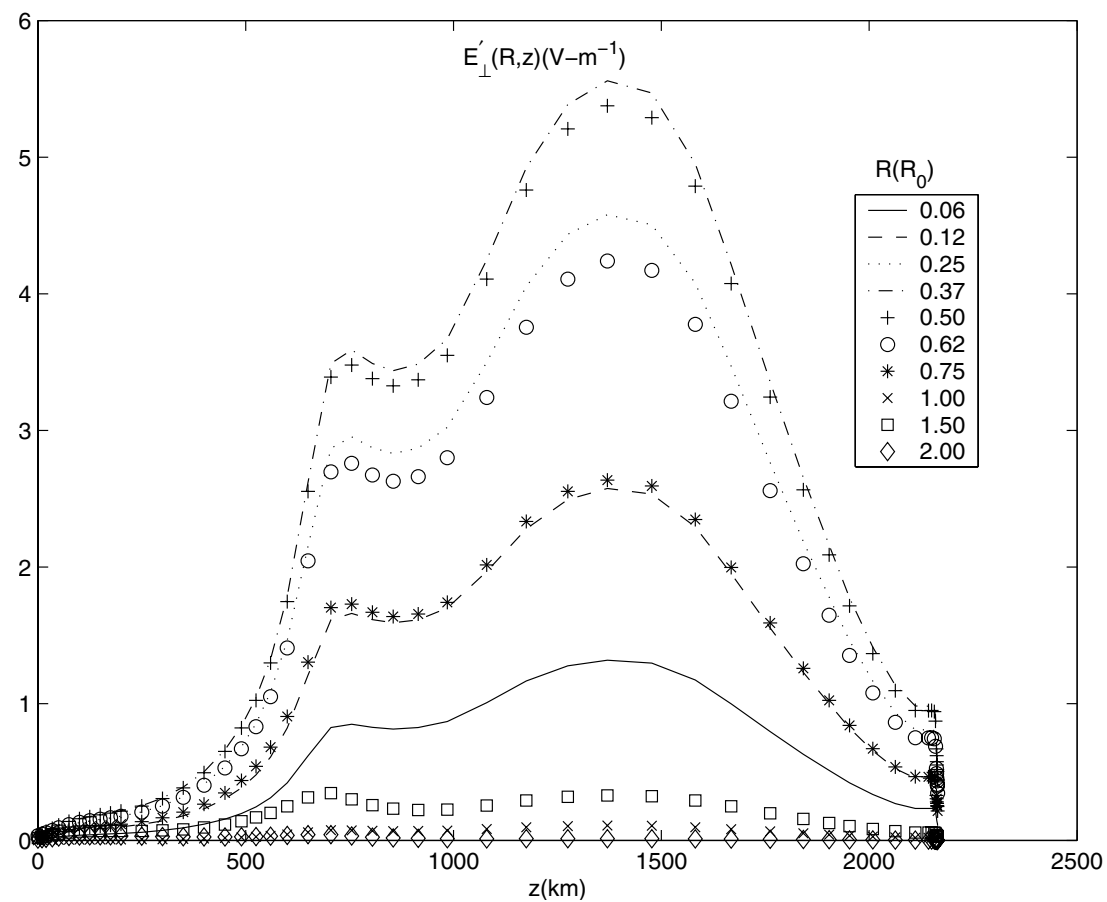

Fig. 11. Magnitude of the component of the CM electric field that is perpendicular to $\boldsymbol{B}$ as a function of $R$ and $z$.

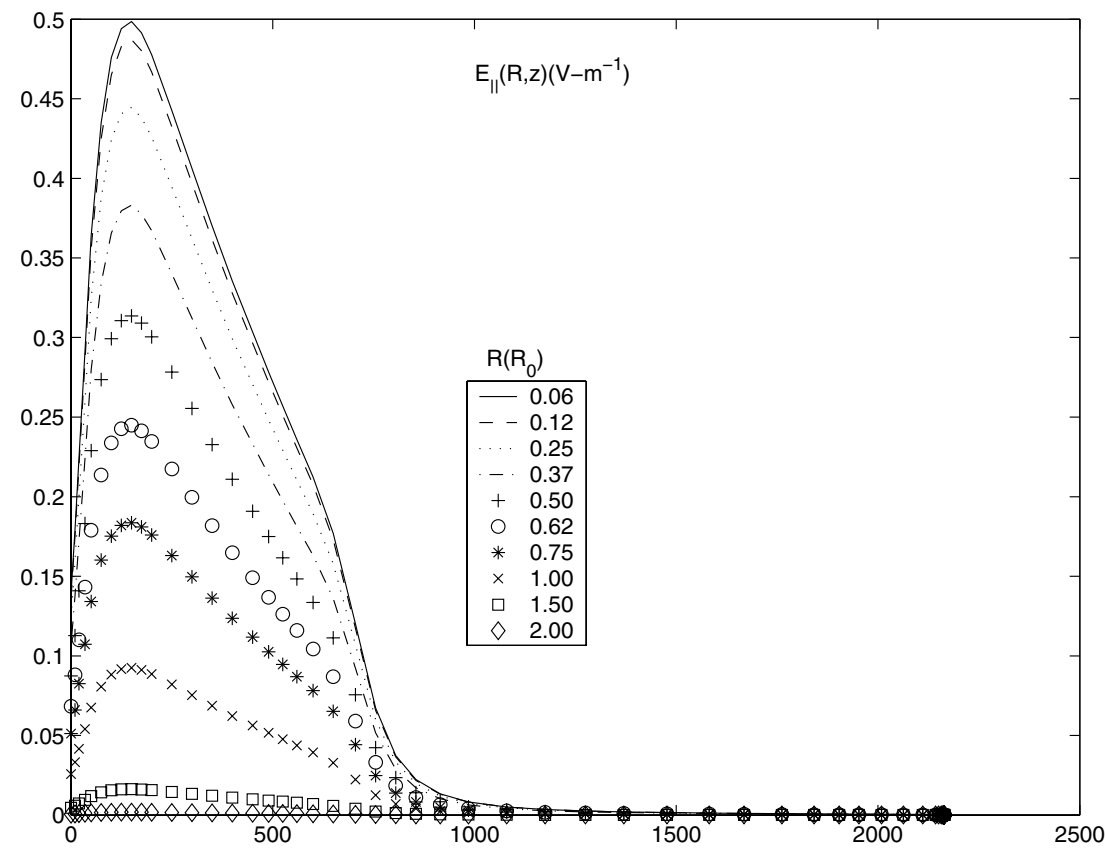

Fig. 12. Magnitude of the component of the electric field that is parallel to $\boldsymbol{B}$ as a function of $R$ and $z$.

dissipation into the upper chromosphere. The results presented here support this proposition.

Figures 18-20 show the parallel, Pedersen, and Hall current densities. $J_{\|} / J \sim 0.9-1$ for $R \leq R_{0}$, and $J_{\|} / J \sim 0.24-0.06$ for $R_{0}<R \leq 2 R_{0}$. The core region, where almost all of the heating occurs, is largely force-free. The plasma rapidly becomes nonforce-free outside the core region.

Although $J_{\|} \gg J_{\perp}$ in the core region, the heating is due almost entirely to Pedersen current dissipation. This may be understood in the following way. From Eqs. (18), (32), (33), (35), and $q_{\|}=J_{\|}^{2} / \sigma_{\|}$it follows that

$$
\frac{q_{\mathrm{P}}}{q_{\|}}=\left(1+\left(\frac{\rho_{\mathrm{n}}}{\rho}\right)^{2} M_{\mathrm{e}} M_{\mathrm{i}, \mathrm{eff}}\right)\left(\frac{J_{\perp}}{J_{\|}}\right)^{2},
$$

where $\rho_{\mathrm{n}} \sim \rho$ in the chromosphere. In the core region, although $\left(J_{\perp} / J_{\|}\right)^{2} \ll 1, M_{\mathrm{e}} M_{\mathrm{i}, \text { eff }}$ is sufficiently large to make $q_{\mathrm{P}} / q_{\|} \gg 1$. This shows that in a strongly magnetized plasma, $q_{\mathrm{P}}$ can be $\gg q_{\|}$even when the magnetic field is largely force-free. 


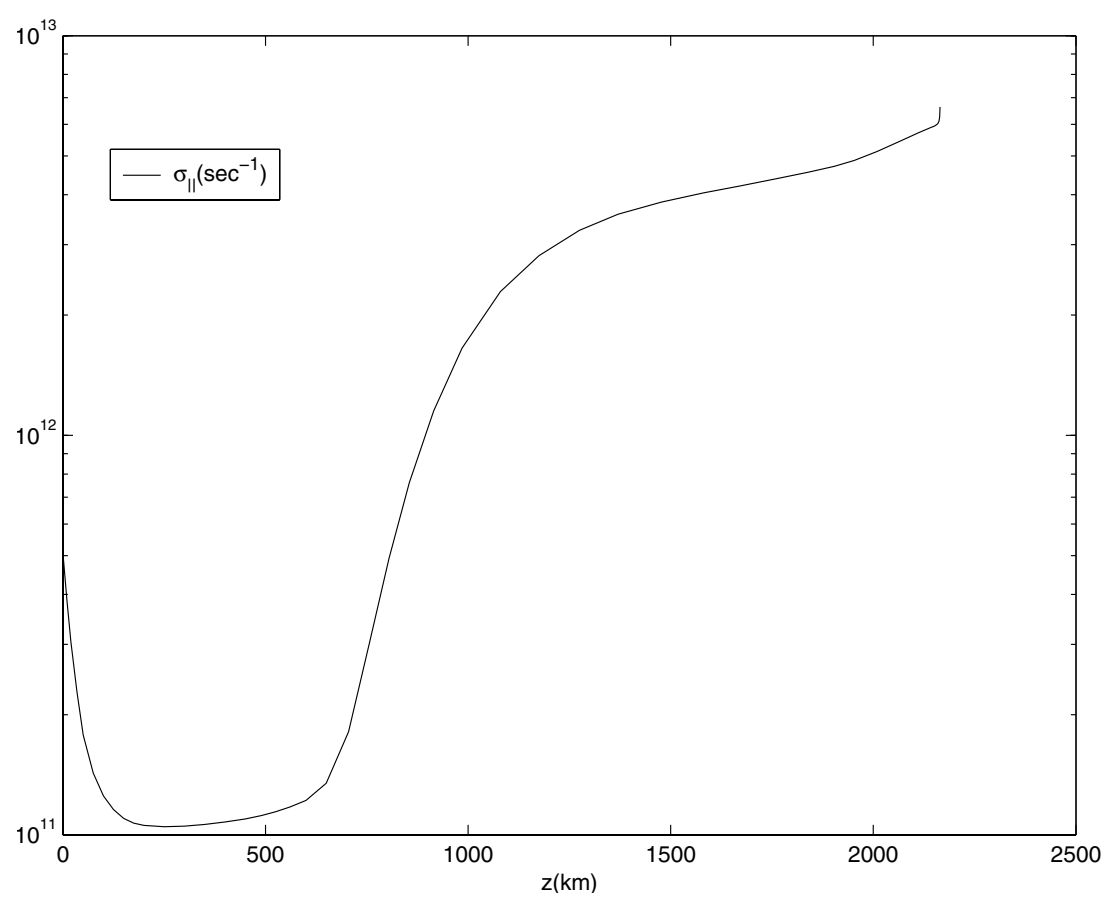

Fig. 13. Parallel conductivity as a function of $z$.

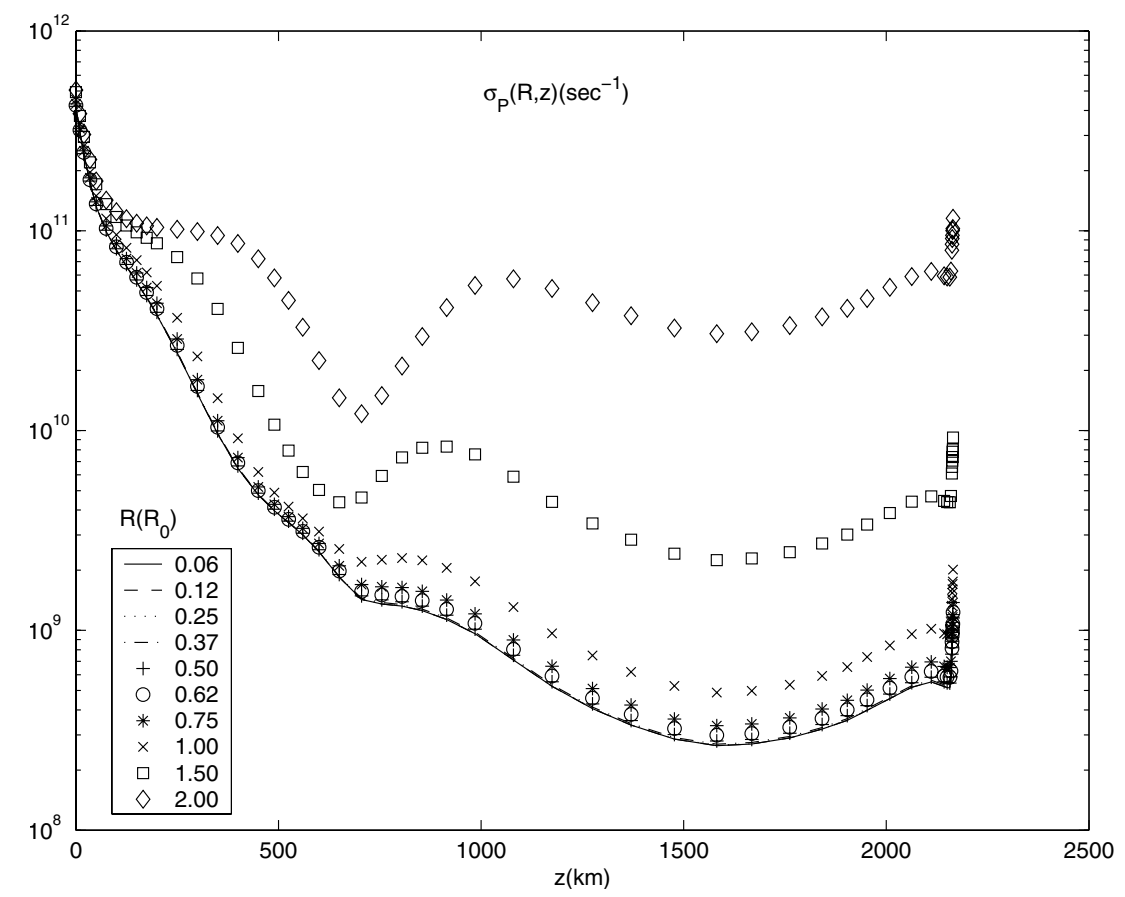

Fig. 14. Pedersen conductivity as a function of $R$ and $z$.

\subsection{Numerical results II}

The solutions for $R^{*}=R_{0, \max }, R_{0, \min }$, and $2 R_{0, \min }$ are similar to the solution for $R^{*}=2 R_{0, \max }$ presented in Sect. 4.1. Some properties of these solutions are briefly discussed here.

For $R^{*}=2 R_{0, \text { min }}$ it is found that $D=4 R_{0, \min }=98.1 \mathrm{~km}$, and $\tau=25.3$. The average heating rates are $F_{2 R_{0 \min }}=4.2 \times$ $10^{6} \mathrm{erg} \mathrm{cm}^{-2} \mathrm{~s}^{-1}, F_{m, 2 R_{0, \text { min }}}=3.4 \times 10^{9} \mathrm{erg} \mathrm{g}^{-1} \mathrm{~s}^{-1}, F_{R_{0, \text { min }}}=$ $1.7 \times 10^{7} \mathrm{erg} \mathrm{cm}^{-2} \mathrm{~s}^{-1}$, and $F_{m, R_{0, \text { min }}}=1.4 \times 10^{10} \mathrm{erg} \mathrm{g}^{-1} \mathrm{~s}^{-1}$. The fraction of $F_{2 R_{0, \text { min }}}$ and $F_{R_{0, \text { min }}}$ due to parallel current dissipation is $\sim 0.03$. The fraction of $F_{m, 2 R_{0, \text { min }}}$ and $F_{m, R_{0, \text { min }}}$ due to parallel current dissipation is $\sim 10^{-5} . J_{\|} / J$ ranges from $\sim 1$ to $\sim 0.68$ for $0 \leq R \leq R_{0}$, and from $\sim 0.47$ to $\sim 0.04$ for $R_{0}<R \leq 2 R_{0}$.

For $R^{*}=R_{0, \text { max }}$ it is found that $D=2 R_{0, \text { max }}=151.8 \mathrm{~km}$, and $\tau=31.6$. The average heating rates are $F_{2 R_{0 \max }}=1.2 \times$ $10^{6} \mathrm{erg} \mathrm{cm}^{-2} \mathrm{~s}^{-1}, F_{m, 2 R_{0, \text { max }}}=9.5 \times 10^{8} \mathrm{erg} \mathrm{g}^{-1} \mathrm{~s}^{-1}, F_{R_{0, \text { max }}}=$ $4.2 \times 10^{6} \mathrm{erg} \mathrm{cm}^{-2} \mathrm{~s}^{-1}$, and $F_{m, R_{0, \text { max }}}=3.4 \times 10^{9} \mathrm{erg} \mathrm{g}^{-1} \mathrm{~s}^{-1, \max }$. The fraction of $F_{2 R_{0, \text { max }}}$ and $F_{R_{0, \text { max }}}$ due to parallel current dissipation 


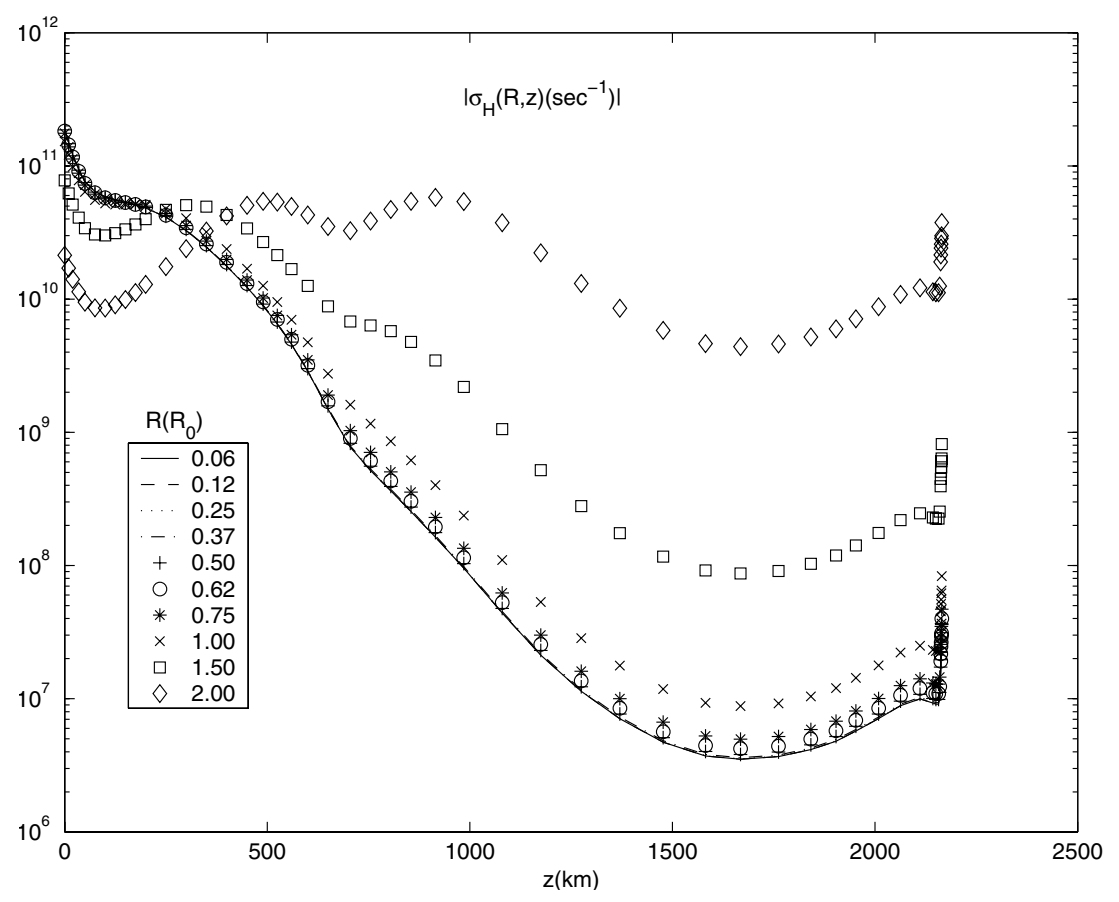

Fig. 15. Magnitude of the Hall conductivity as a function of $R$ and $z$.

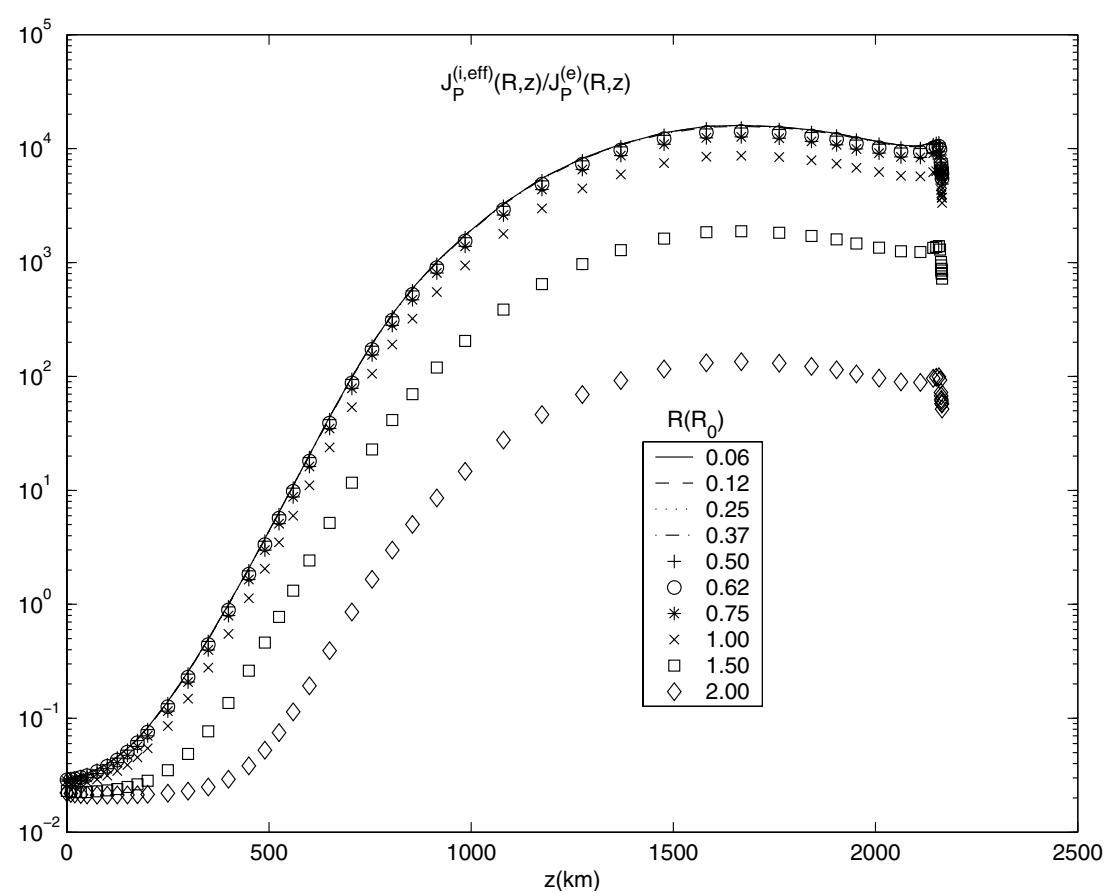

Fig. 16. Ratio of the magnitudes of the effective ion and electron Pedersen current densities as a function of $R$ and $z$.

is $\sim 0.05$. The fraction of $F_{m, 2 R_{0, \max }}$ and $F_{m, R_{0, \max }}$ due to parallel current dissipation is $\sim 10^{-3} . J_{\|} / J$ ranges from $\sim 1$ to $\sim 0.9$ for $0 \leq R \leq R_{0}$, and from $\sim 0.24$ to $\sim 0.05$ for $R_{0}<R \leq 2 R_{0}$.

For $R^{*}=R_{0, \text { min }}$ it is found that $D=2 R_{0, \text { min }}=97.5 \mathrm{~km}$, and $\tau=12.7$. The average heating rates are $F_{2 R_{0, \min }}=1.1 \times$ $10^{6} \mathrm{erg} \mathrm{cm}^{-2} \mathrm{~s}^{-1}, F_{m, 2 R_{0, \text { min }}}=8.6 \times 10^{8} \mathrm{erg} \mathrm{g}^{-1} \mathrm{~s}^{-1}, F_{R_{0, \text { min }}}=$ $4.2 \times 10^{6} \mathrm{erg} \mathrm{cm}^{-2} \mathrm{~s}^{-1}$, and $F_{m, R_{0, \min }}=3.4 \times 10^{9} \mathrm{erg} \mathrm{g}^{-1} \mathrm{~s}^{-1}$. The fraction of $F_{2 R_{0, \min }}$ and $F_{R_{0, \min }}$ due to parallel current dissipation is $\sim 0.03$. The fraction of $F_{m, 2 R_{0, \text { min }}}$ and $F_{m, R_{0, \text { min }}}$ due to parallel current dissipation is $\sim 10^{-3} . J_{\|} / J$ ranges from $\sim 1$ to $\sim 0.68$ for $0 \leq R \leq R_{0}$, and from $\sim 0.47$ to $\sim 0.04$ for $R_{0}<R \leq 2 R_{0}$.

As in the case of the solution in Sect. 4.1, the core region for these solutions is largely force-free, the plasma rapidly becomes non-force-free outside this region, and the magnetic field is highly twisted in that $B_{\theta} \gg B_{R}$. The heating rate in the core region is $\sim 10-100$ times larger than outside this region. Almost all of the heating occurs in the largely force-free core plasma, and is due to Pedersen current dissipation. The 


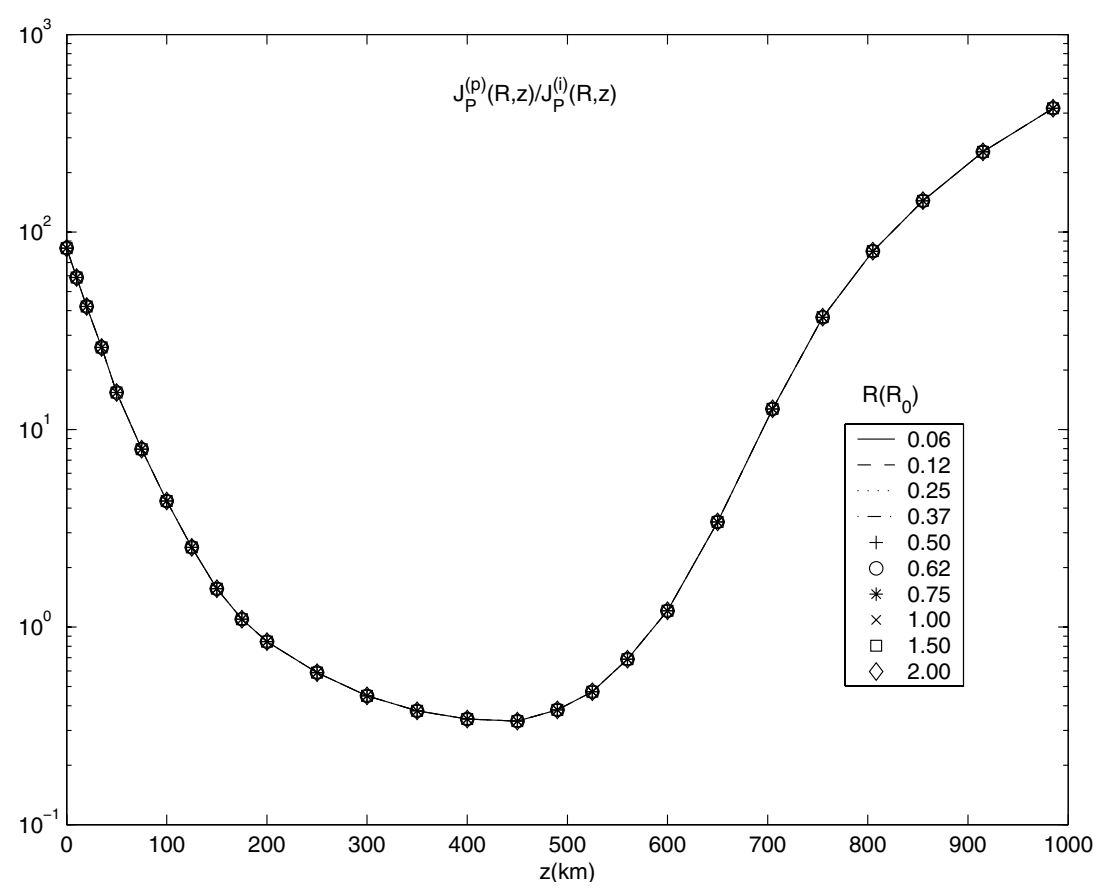

Fig. 17. Ratio of the magnitudes of the proton and proxy ion Pedersen current densities as a function of $R$ and $z$.

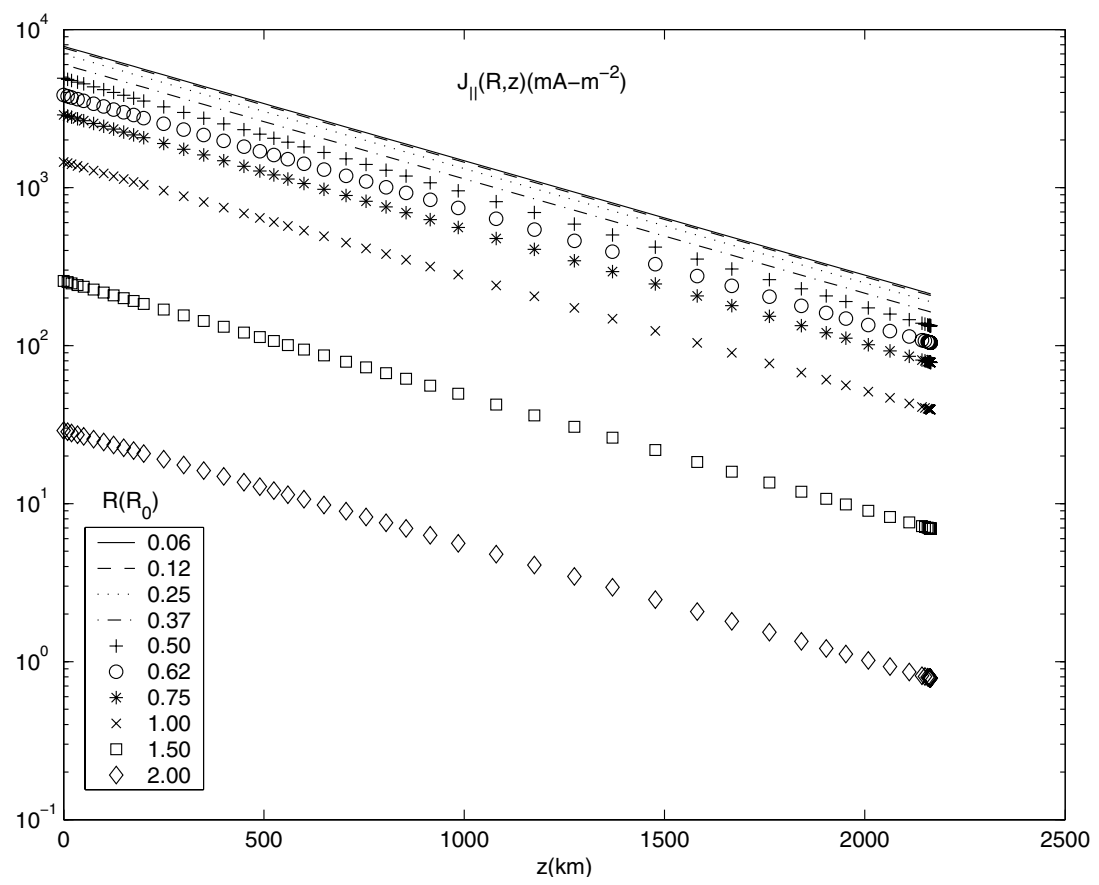

Fig. 18. Current density parallel to $\boldsymbol{B}$ as a function of $R$ and $z$.

range of diameters of the core regions for the four solutions is $\sim 49-152 \mathrm{~km}$, so essentially all of the heating occurs on subresolution horizontal scales.

\section{The model - Part 2}

The model presented in Sect. 2 does not predict $\boldsymbol{V}$ or $\boldsymbol{E}_{\perp}$. It is necessary to know these quantities in order to determine the contributions of $\boldsymbol{E}_{\perp}$ and $\boldsymbol{E}_{\mathrm{c}}$ to $\boldsymbol{E}^{\prime}$.

Here the model in Sect. 2 is extended to compute $\boldsymbol{V}$ and $\boldsymbol{E}_{\perp}$, and estimate the $R$ dependence of the total pressure and density $p$ and $\rho$, which in FAL CM only depend on $z$.

\subsection{Momentum conservation equation}

Assuming a steady state that only depends on $R$ and $z$, and ignoring viscosity and the convection term $\boldsymbol{V} \cdot \nabla \boldsymbol{V}$, the momentum equation is

$\nabla \mathrm{p}=\frac{\boldsymbol{J} \times \boldsymbol{B}}{c}-\rho g \hat{z}$

$g=2.74 \times 10^{4} \mathrm{~cm} \mathrm{~s}^{-2}$ is the gravitational acceleration at the photosphere, and $\hat{z}$ is a unit vector in the vertical direction. 


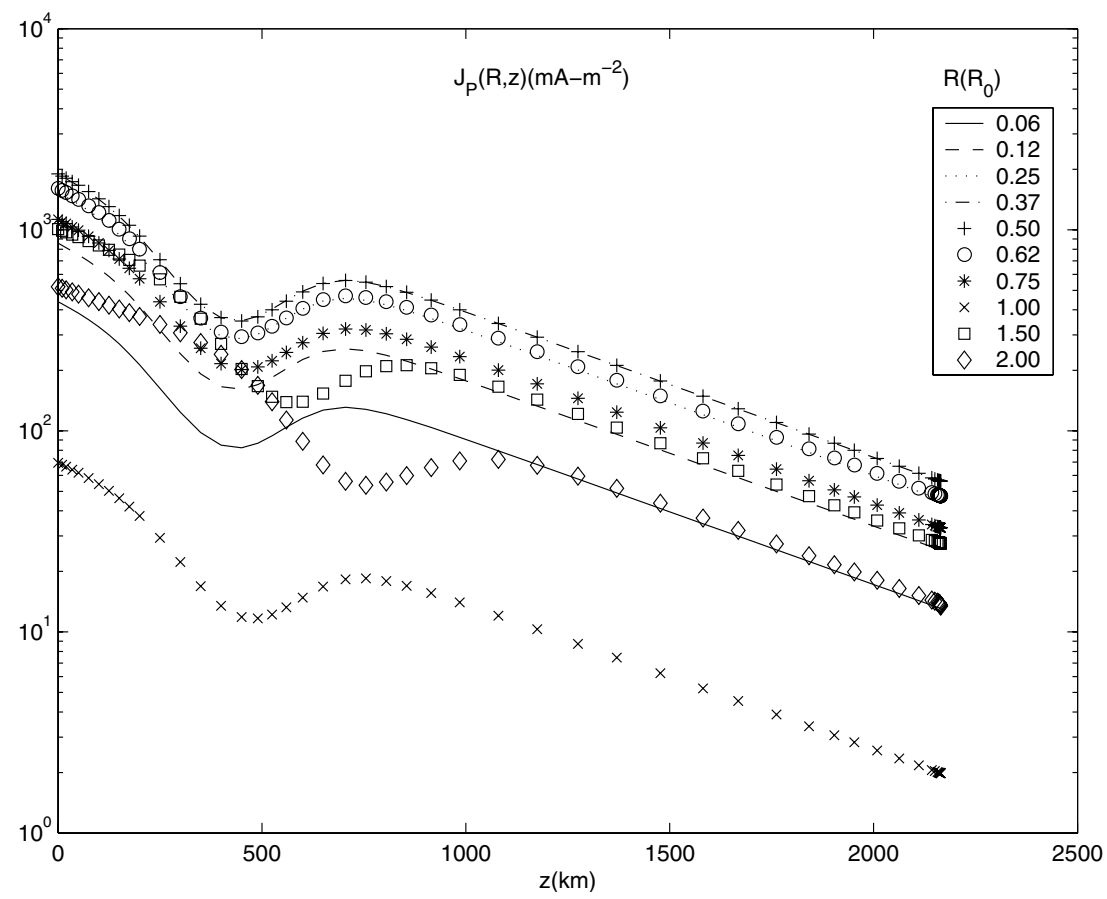

Fig. 19. Magnitude of Pedersen current density as a function of $R$ and $z$.

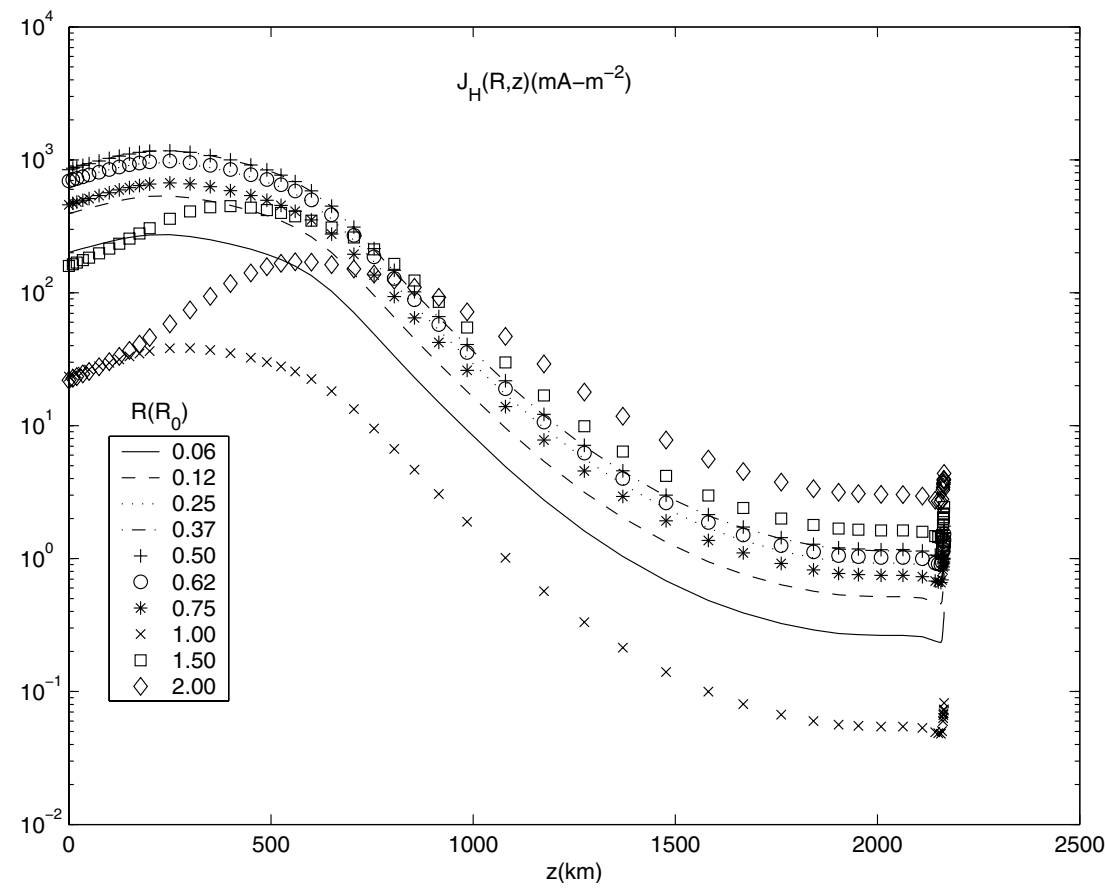

Fig. 20. Magnitude of Hall current density as a function of $R$ and $z$.

The $\theta$ component of Eq. (53) is identical to Eq. (5) that is used to determine $B_{\theta}$. This equation connects the two parts of the model.

Using $\boldsymbol{B}$ from Sect. 2, the $R$ and $z$ components of Eq. (53) are solved for $p$ and $\rho$, giving

$$
\begin{aligned}
p(R, z)= & \mathrm{e}^{\left(-2 z / L_{B}\right)}\left\{p_{0}+\frac{B_{0}^{2}-b^{2}}{8 \pi}-\frac{B_{0}^{2}}{8 \pi} \frac{R_{0}^{2}\left(1+\tau^{2}\right)}{8 L_{B}^{2}}\right. \\
& \left.\times\left(1-\mathrm{e}^{-2 R^{2} / R_{0}^{2}}\right)\right\}
\end{aligned}
$$

and

$$
\begin{aligned}
\rho(R, z)= & \frac{2}{L_{B} g}\left\{p(R, z)+\frac{B_{R}^{2}}{8 \pi}\left(1+\tau^{2}+\left(\frac{2 L_{B}}{R_{0}}\right)^{2}\right.\right. \\
& \left.\left.\times\left(\frac{R^{2}}{R_{0}^{2}}-2\right)\right)\right\}
\end{aligned}
$$

where $p_{0} \equiv p(0,0)$ and $b(R)=\mathrm{e}^{\left(z / L_{B}\right)} B$. The total number density $n(R, z)$ is obtained from $\rho$ using the relation $n=\rho / m_{0}$ where $m_{0}=1.2763 m_{\mathrm{p}}$ is the average particle mass computed as the number density weighted average particle mass 


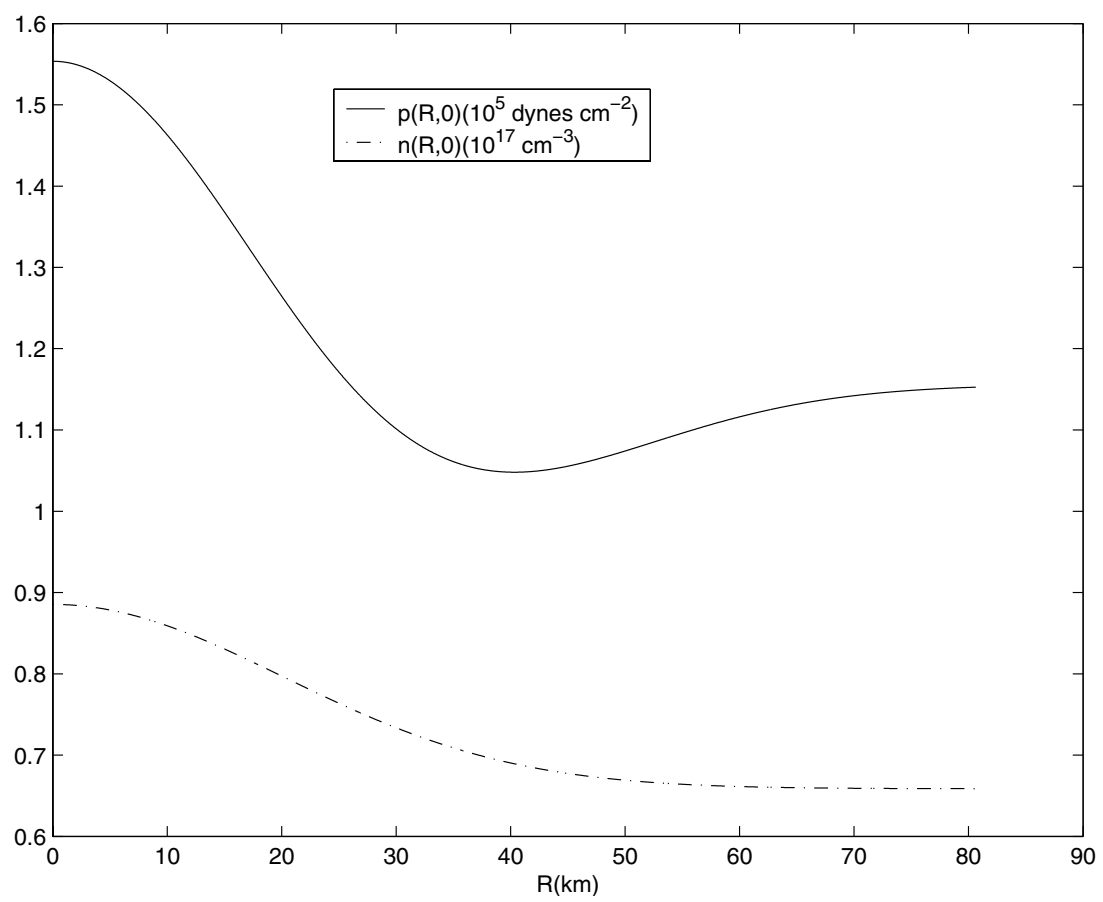

Fig. 21. Pressure and number density as functions of $R$ at $z=0$. The pressure is given in units of $10^{5}$ dynes $\mathrm{cm}^{-2}$. The number density is given in units of $10^{17} \mathrm{~cm}^{-3}$.

at $z=0$ given by FAL CM. Require $p(\infty, 0)=p_{\text {FAL }}$ where $p_{\mathrm{FAL}}=\left(n_{\mathrm{e}}(0)+n_{\mathrm{p}}(0)+n_{\mathrm{H}}(0)+n_{\mathrm{He}}(0)+n_{\mathrm{He} \mathrm{II}}(0)+n_{\mathrm{He} \mathrm{III}}(0)+\right.$ $\left.n_{\mathrm{i}}(0)\right) k_{\mathrm{B}} T(0) . k_{\mathrm{B}}$ is Boltzmann's constant, and $T$ is the FAL CM temperature. This is a realistic requirement if the magnetic structures, assumed to be of radius $2 R_{0}$, are separated by distances $d$ such that $\exp \left(-d^{2} / R_{0}^{2}\right) \ll 1$. For $f=0.02$ one has $d \sim 3.54 R_{0}$, in which case $\exp \left(-d^{2} / R_{0}^{2}\right) \sim 10^{-6}$. Then

$p_{0}=p_{\mathrm{FAL}}+\frac{B_{0}^{2}}{8 \pi}\left(\frac{R_{0}^{2}\left(1+\tau^{2}\right)}{8 L_{B}^{2}}-1\right)$.

Since $p_{\text {FAL }}=1.1557 \times 10^{5}$ dynes $\mathrm{cm}^{-2}$ it follows that $p_{0} \sim$ $1.5536 \times 10^{5}$ dynes $\mathrm{cm}^{-2}$, and $n(0,0) \sim 8.8536 \times 10^{16} \mathrm{~cm}^{-3}$.

Figure 21 shows $p(R, 0)$ and $n(R, 0)$. The pressure and density in the core region are larger than in the surrounding relatively field free region. For $R \geq 2 R_{0}$ they are essentially equal to their asymptotic values $p(\infty, 0)=p_{\mathrm{FAL}}$ and $n(\infty, 0)=$ $2 p(\infty, 0) / L_{B} m_{0} g \sim 6.5861 \times 10^{16} \mathrm{~cm}^{-3}$. The pressure and density on the axis $R=0$ are only $\sim 35 \%$ larger than their values in the field free plasma.

\subsection{Determination of $V_{R}$ and $V_{z}$}

The mass conservation equation is

$\nabla \cdot(\rho \boldsymbol{V})=0$

which may be written as

$\frac{1}{R} \frac{\partial\left(R \rho(R, 0) V_{R}\right)}{\partial R}-\frac{2 \rho(R, 0) V_{z}}{L_{B}}+\rho(R, 0) \frac{\partial V_{z}}{\partial z}=0$.

Equation (58) is simplified using the following approximation. It is expected, and later verified that $\boldsymbol{V}$ is mainly parallel to $\boldsymbol{B}$. Then $\rho \boldsymbol{V} \sim A \boldsymbol{B}$, where $A$ must be constant along magnetic field lines. In general, $A$ may vary with $R$ and $z$. Here the simplest case of $A$ independent of $R$ and $z$ is considered. Then from $V_{z} \sim A B_{z} / \rho$ it follows that $\partial V_{z} / \partial z \sim V_{z} / L_{B}$, in which case Eq. (58) becomes

$\frac{1}{R} \frac{\partial\left(R \rho(R, 0) V_{R}\right)}{\partial R}-\frac{\rho(R, 0) V_{z}}{L_{B}}=0$,

with the boundary condition $V_{R}(0, z)=0$.

$\boldsymbol{E}^{\prime}$ is known from Sect. 2.2, and $E_{\theta}=0$ since $\nabla \times \boldsymbol{E}=0$. Then the $\theta$ component of $\boldsymbol{E}^{\prime}=\boldsymbol{E}+(\boldsymbol{V} \times \boldsymbol{B}) / c$ gives

$V_{z}=\frac{\left(c E_{\theta}^{\prime}+V_{R} B_{z}\right)}{B_{R}}$.

Equation (60) is used to eliminate $V_{z}$ in Eq. (59). Then for each value of $z$, Eq. (59) is solved for $V_{R} . V_{z}$ is then determined by Eq. (60).

\subsection{Determination of $\boldsymbol{E}$ and $V_{\theta}$}

The equation $\nabla \times \boldsymbol{E}=0$ reduces to $E_{\theta}=0$ and

$\frac{\partial E_{R}}{\partial z}-\frac{\partial E_{z}}{\partial R}=0$.

It is now assumed that $\partial \boldsymbol{E} / \partial z \sim-\boldsymbol{E} / L_{B}$.

Multiplying the $R$ component of $\boldsymbol{E}^{\prime}=\boldsymbol{E}+(\boldsymbol{V} \times \boldsymbol{B}) / c$ by $B_{R}$, the $z$ component by $B_{z}$, adding the two resulting equations, and using Eq. (61) to express $E_{R}$ in terms of $\partial E_{z} / \partial R$ gives the following equation for $E_{z}$.

$$
\begin{aligned}
\frac{\partial E_{z}}{\partial R} & +\frac{1}{L_{B} B_{R}}\left\{B_{R} E_{R}^{\prime}+B_{z} E_{z}^{\prime}-B_{z} E_{z}-\frac{B_{\theta}}{c}\right. \\
& \left.\times\left(V_{R} B_{z}-V_{z} B_{R}\right)\right\}=0
\end{aligned}
$$




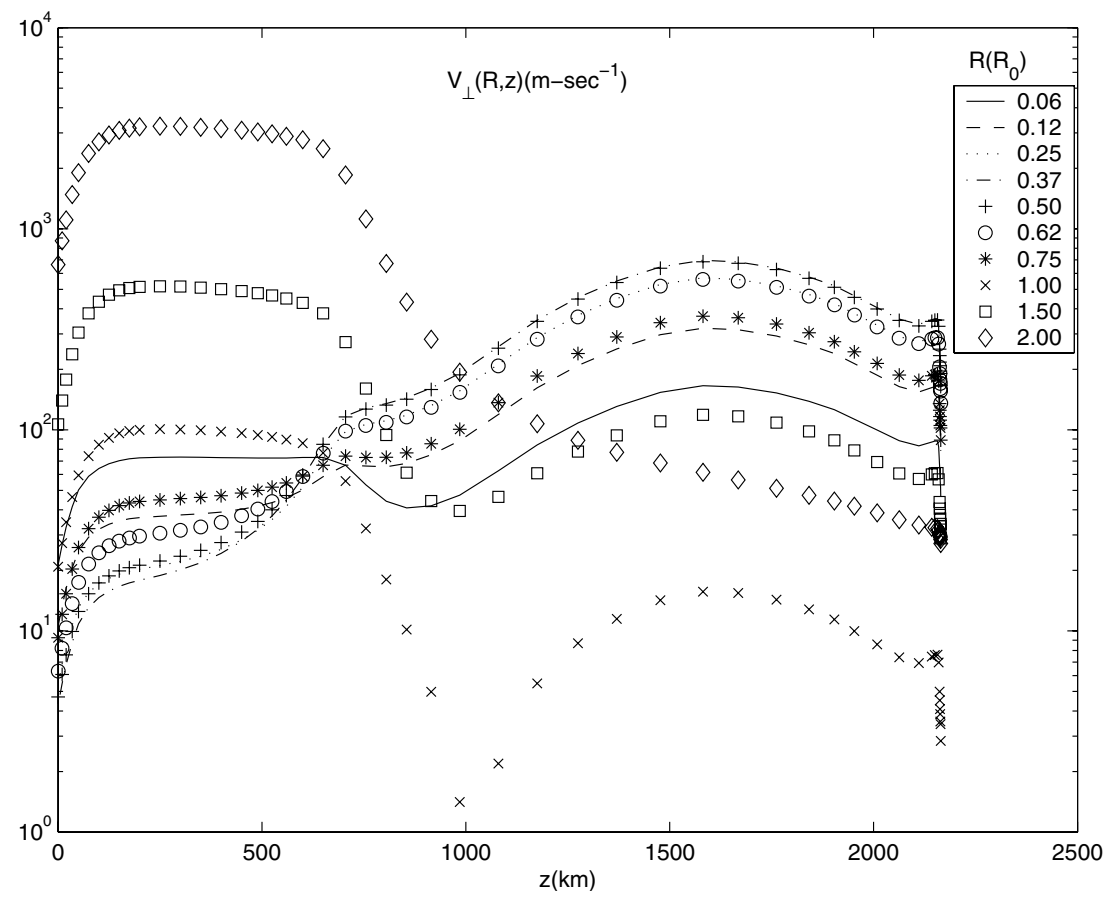

Fig. 22. Magnitude of the flow velocity perpendicular to $\boldsymbol{B}$ as a function of $R$ and $z$.

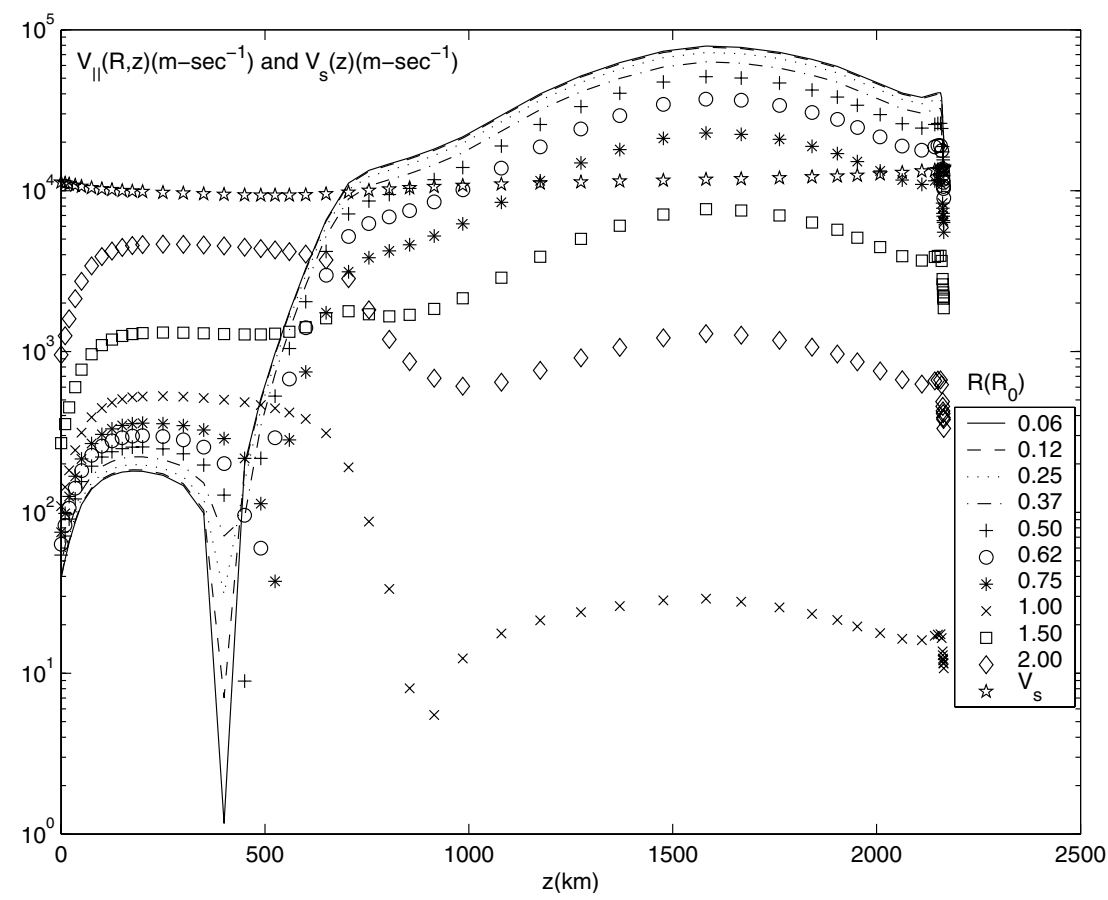

Fig. 23. Magnitude of the flow velocity parallel to $\boldsymbol{B}$ as a function of $R$ and $z$.

with the boundary condition

$E_{z}(0, z)=\frac{J_{z}(0, z)}{\sigma_{\|}(0, z)}$

For each value of $z$, Eq. (62) is solved for $E_{z}(R, z)$.

The $z$ component of $\boldsymbol{E}^{\prime}=\boldsymbol{E}+(\boldsymbol{V} \times \boldsymbol{B}) / c$ then determines $V_{\theta}$ by

$V_{\theta}=\frac{\left(c\left(E_{z}-E_{z}^{\prime}\right)+V_{R} B_{\theta}\right)}{B_{R}}$
$E_{R}$ is obtained from Eq. (61) as

$E_{R}=-L_{B} \frac{\partial E_{z}}{\partial R}$

where $\partial E_{z} / \partial R$ is given by Eq. (62).

Figures 22 and 23 show $V_{\perp}$ and $V_{\|} \cdot V_{\|} \sim 10-100 V_{\perp}$ throughout most of the photosphere and chromosphere. $V_{\|}$exceeds the sound speed by up to a factor $\sim 7$ in the chromosphere. $V_{\perp} \lesssim 4 \mathrm{~km} \mathrm{~s}^{-1}$ in the photosphere, and is $\$ 700 \mathrm{~m} \mathrm{~s}^{-1}$ in the 


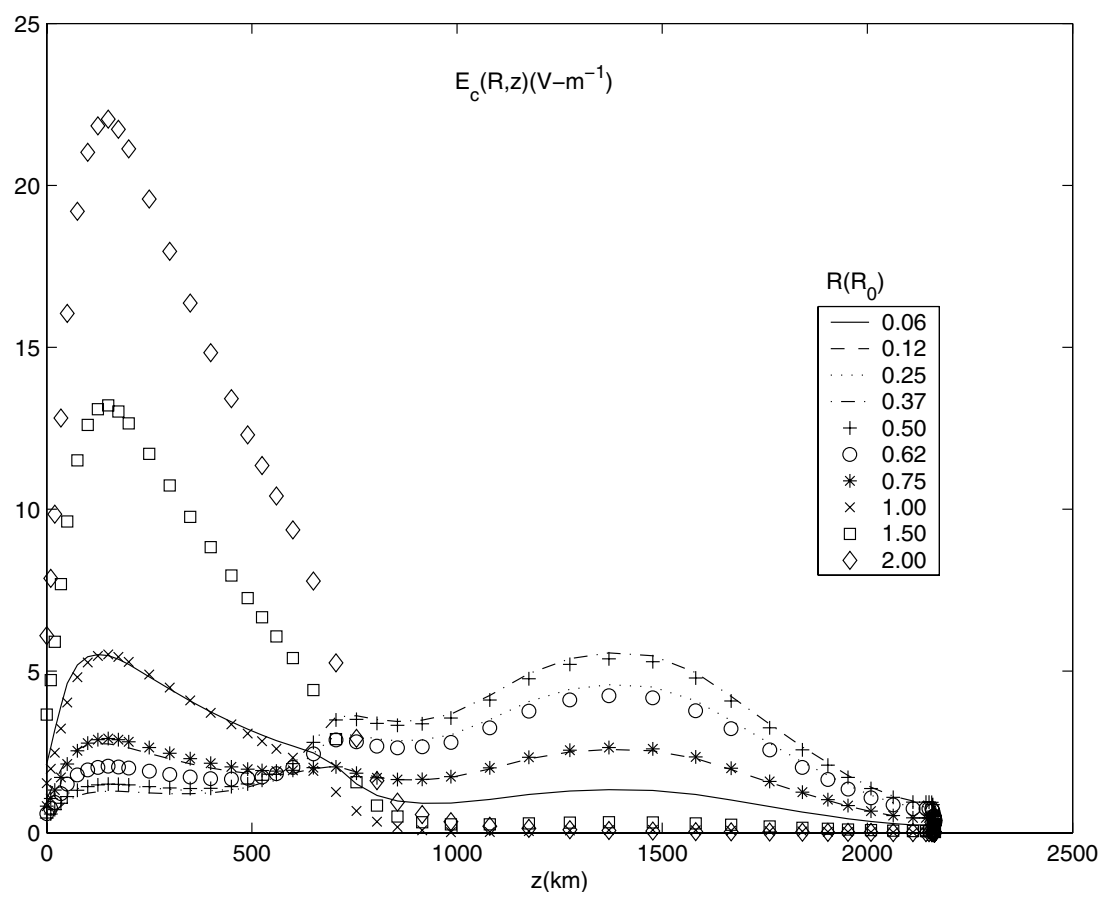

Fig. 24. Magnitude of the convection electric field as a function of $R$ and $z$.

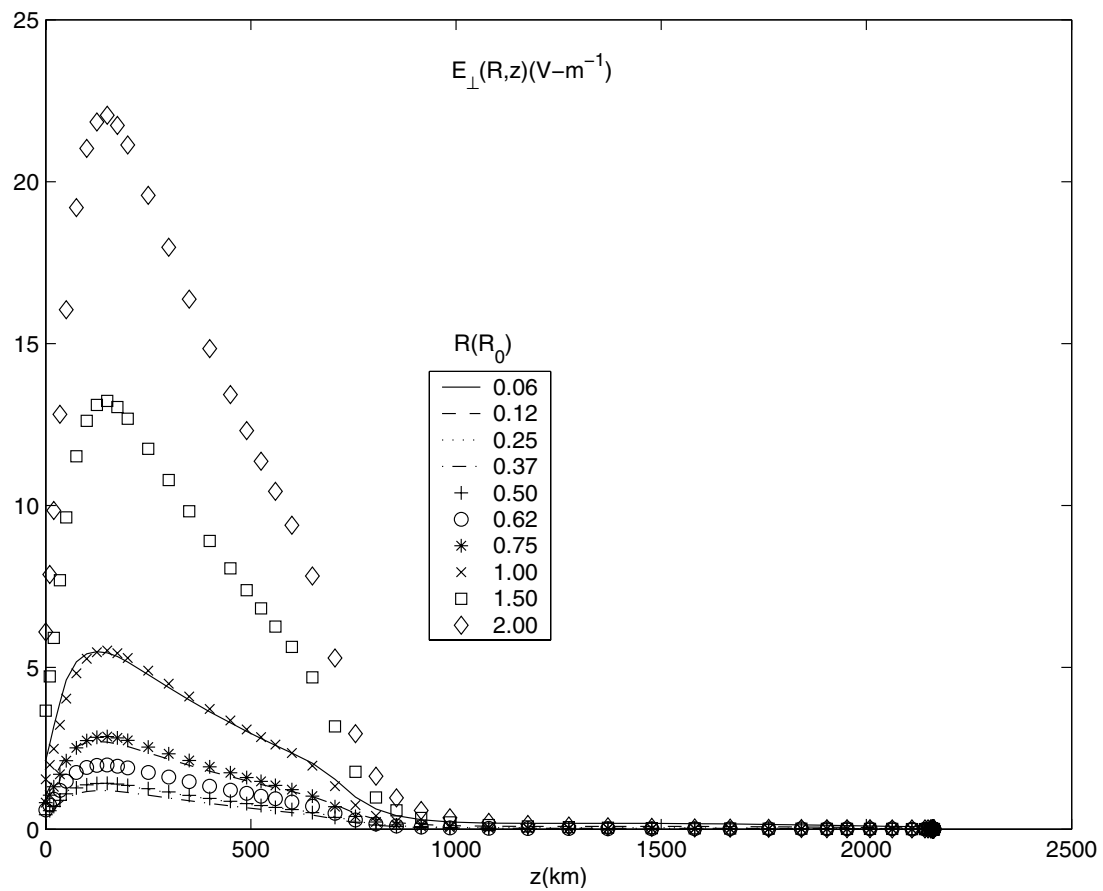

Fig. 25. Magnitude of the component of $\boldsymbol{E}$ perpendicular to $\boldsymbol{B}$ as a function of $R$ and $z$.

chromosphere. $V_{\perp}$ generates the convection electric field that drives the Pedersen current.

Figures 24-26 show $E_{\mathrm{c}}, E_{\perp}$, and $E_{\mathrm{c}} / E_{\perp} . E_{\mathrm{c}} \gg E_{\perp}$ essentially everywhere in the chromosphere except for $R \sim 2 R_{0}$. $E_{\mathrm{c}}$ rapidly dominates $E_{\perp}$ beginning near $z_{T \text { min }} . \boldsymbol{E}_{\mathrm{c}}$ and $\boldsymbol{E}_{\perp}$ largely cancel in the photosphere in that $E_{\perp}^{\prime} \ll E_{\perp}$. The Pedersen current and the associated resistive heating in the chromosphere are driven by the convection electric field.
The rate at which the electric field transfers energy to the fluid is

$$
\begin{aligned}
\boldsymbol{J} \cdot \boldsymbol{E} & =\boldsymbol{J} \cdot\left(\boldsymbol{E}_{\|}+\boldsymbol{E}_{\perp}^{\prime}\right)+\boldsymbol{V} \cdot(\boldsymbol{J} \times \boldsymbol{B}) / c \\
& =q+\boldsymbol{V} \cdot(\boldsymbol{J} \times \boldsymbol{B}) / c .
\end{aligned}
$$

The first term on the right hand side of Eq. (67) is the total resistive heating rate. The second term is the rate of change of 


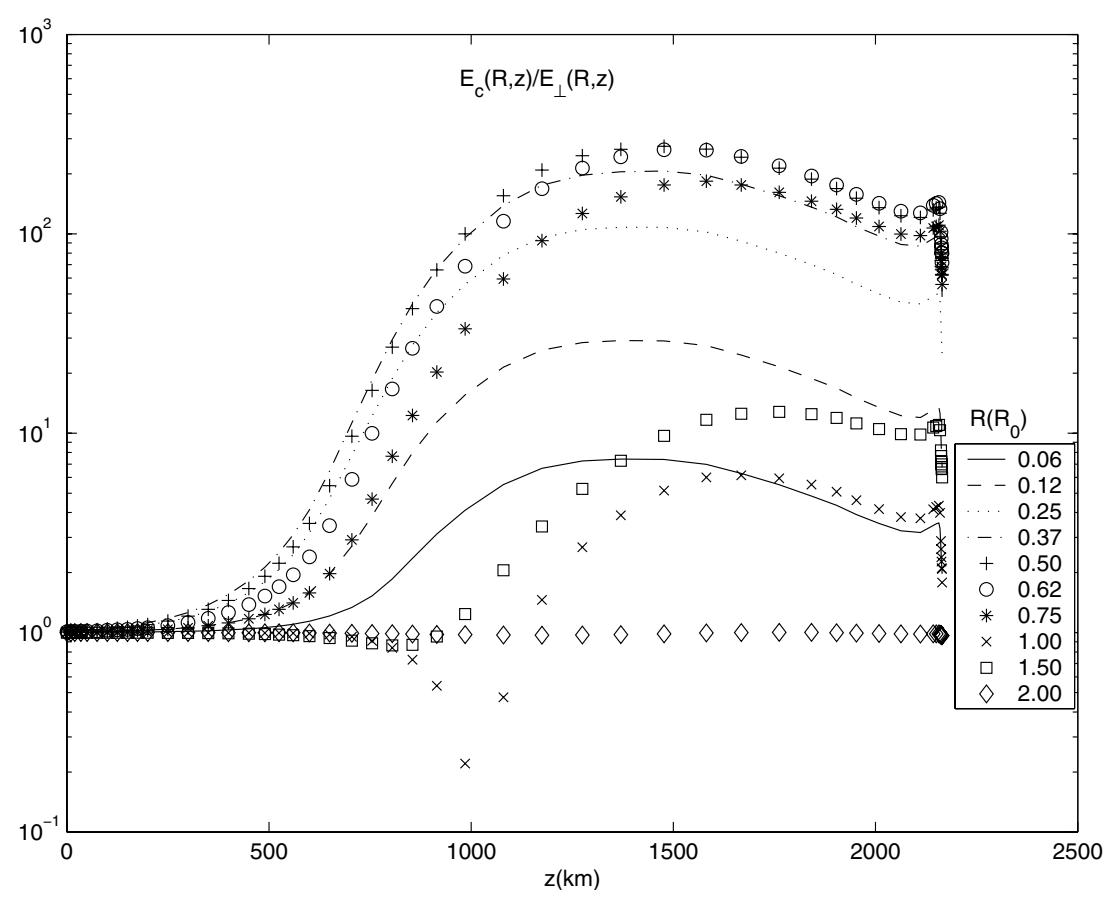

Fig. 26. Ratio of the magnitudes of the convection electric field and $\boldsymbol{E}_{\perp}$ as a function of $R$ and $z$.

the kinetic energy of the CM of the fluid. If heating is mainly driven by the convection electric field then $q \sim q_{\mathrm{P}}$, and

$\boldsymbol{J} \cdot \boldsymbol{E} \sim \boldsymbol{J} \cdot(\boldsymbol{V} \times \boldsymbol{B}) / c+\boldsymbol{V} \cdot(\boldsymbol{J} \times \boldsymbol{B}) / c=0$,

in which case there is little exchange of energy between $\boldsymbol{E}$ and the plasma. The exchange of energy is mainly between the kinetic energy of the bulk flow, and the current density. The magnetic field enables and mediates a direct transfer of kinetic energy from the bulk flow to the current density. This energy is thermalized by collisions. Convection across magnetic field lines directly drives resistive heating by Pedersen current dissipation. The thermally driven convection in the convection zone is a continuous source of mechanical energy for driving this heating process. The solar chromosphere acts as an MHD power generator converting the energy of thermal convection into that of electric currents that are dissipated by the resistive load of the plasma.

In the MHD wave heating model of Goodman (2000) it is found that $\boldsymbol{E}_{\perp}^{\prime} \sim \boldsymbol{E}_{\mathrm{c}}, E_{\mathrm{c}} \sim 0.3 \mathrm{~V} \mathrm{~m}^{-1}$, and $V_{\perp} \sim 17-21 \mathrm{~m} \mathrm{~s}^{-1}$ at $z \sim 750 \mathrm{~km}$. That model does not take account of the filling factor of the magnetic flux tubes in which the heating is found to occur. The effect of including a filling factor is estimated as follows. If $q_{\mathrm{P}}$ is driven by a convection electric field then $q_{\mathrm{P}} \sim f \sigma_{\mathrm{P}}\left(V_{\perp} B / c\right)^{2}$. Then for given $\left(q_{\mathrm{P}}, B, \sigma_{\mathrm{P}}\right)$, $V_{\perp}$ and $E_{\perp}^{\prime}$ are $\propto f^{-1 / 2}$. Then for $f=0.02$ the values of $E_{\perp}^{\prime}$ and $V_{\perp}$ just stated are changed to $E_{\perp}^{\prime} \sim 2.1 \mathrm{~V} \mathrm{~m}^{-1}$, and $V_{\perp} \sim 120-149 \mathrm{~m} \mathrm{~s}^{-1}$. These values are comparable to the values of $E_{\perp}^{\prime}$ and $V_{\perp}$ at $z=750 \mathrm{~km}$ in Figs. 11 and 22. In the model in Goodman (2000) there is no flow in the equilibrium state, and the equilibrium magnetic field is constant, and hence unbounded and current free. Then $\boldsymbol{E}_{\perp}^{\prime}$ and $\boldsymbol{V}_{\perp}$ are entirely due to the MHD wave. By contrast, the model considered here assumes a steady state with flow, and a localized, non-potential magnetic field. The fact that estimates of $E_{\perp}^{\prime}$ and $V_{\perp}$ are similar for these two models at $z \sim 750 \mathrm{~km}$ for the same value of $f$, and for the requirement that the resistive heating rate, which is essentially $q_{\mathrm{P}}$, match that predicted by FAL CM follows from the fact that $\sigma_{\mathrm{P}}, B$, and $q_{\mathrm{P}}$ have similar values in both models at that height.

\section{Conclusions}

As observational resolution increases, the observed filling factor of magnetic field structures in the photosphere increases, including structures with kilogauss magnetic field strengths. This trend, together with the model results reported here and in Goodman $(2000,2001,2004)$ lead to the proposition that the chromospheres of the Sun and other solar type stars are created by Pedersen current dissipation driven by a convection electric field in magnetic structures with horizontal spatial dimensions that are currently sub-resolution. This is an MHD power generation mechanism. Mechanical work is done to drive a CM flow of plasma across magnetic field lines. The resulting convection electric field drives current against the resistive load of the plasma, generating thermal energy. Observationally, resolution of the photospheric magnetic field on spatial scales $\lesssim 0.1^{\prime \prime}$ is necessary in order to resolve the core regions of the magnetic structures in which almost all of the heating occurs. The essential difference between network and internetwork chromospheric heating is that the filling factor of the sub-resolution magnetic structures in which the heating occurs is larger in the network than in the internetwork.

The proposed heating mechanism is consistent with observational and semi-empirical model evidence that most of the magnetic flux through the photosphere is in the form of magnetic field concentrations with sub-resolution diameters as small as a few kilometers, and field strengths $\sim 10^{2}-10^{3} \mathrm{G}$ (Sánchez Almeida \& Landi degl'Innocenti 1996; 
Sánchez Almeida et al. 1996; Sánchez Almeida 1997, 2000; Sánchez Almeida \& Lites 2000; Sánchez Almeida et al. 2001; Socas-Navarro \& Sánchez Almeida 2002; Domínguez Cerdeña et al. 2003). A common view is that the internetwork chromosphere is heated by acoustic wave dissipation, and that magnetic field related heating mechanisms may only be important in the network. Judge et al. (2003) question this view, and present observational and modeling evidence that the solar upper chromospheric internetwork is heated by a magnetic field related mechanism. This is consistent with the proposition that the entire chromosphere outside of flaring regions is heated by an MHD mechanism of the type described in this paper.

It is the increase of the particle magnetizations with height from values $\ll 1$ to values $>1$ in the weakly ionized photospheric plasma that transforms Pedersen current dissipation into a qualitatively new, and highly efficient heating mechanism, and, in the presence of the $\mathrm{CM}$ convection across magnetic field lines that exists everywhere and always, simultaneously causes the buildup of the magnitude of the convection electric field to a value large enough to increase $q_{\mathrm{P}}$ to a value sufficiently large to cause a temperature inversion and create the chromosphere. The main features of this process are summarized as follows.

A temperature inversion cannot occur in a weakly magnetized atmosphere: collisional momentum transfer to charged particles in the weakly ionized photosphere is dominated by charged particle $-\mathrm{H}$ collisions. The corresponding reduced collision frequencies are much larger than the cyclotron frequencies. Then the charged particles are unmagnetized, the conductivity tensor is essentially isotropic, and the conductivity in any direction is $\sim \sigma_{\|}^{(\mathrm{e})}$. In particular, $\sigma_{\mathrm{P}} \sim \sigma_{\|}^{(\mathrm{e})}$. The conductivity is high enough so that the $\mathrm{CM}$ electric field is sufficiently shorted out to prevent its magnitude from building up to a value sufficiently large to cause $q_{\mathrm{P}}$ to become large enough to cause a temperature inversion. This shorting out of the CM electric field implies that $\boldsymbol{E}_{\perp}$ and $\boldsymbol{E}_{\mathrm{c}}$ largely cancel in that $E_{\perp}^{\prime} / E_{\mathrm{c}} \equiv\left|\boldsymbol{E}_{\perp}+\boldsymbol{E}_{\mathrm{c}}\right| / E_{\mathrm{c}} \ll 1 . M_{s} \propto B T^{1 / 2} / n_{\mathrm{H}}$ in the weakly ionized photosphere. If $B T^{1 / 2}$ decreases with height faster than $n_{\mathrm{H}}$ then $M_{s}$ remains $\ll 1$, and there is no chromosphere.

Magnetization increases with height in solar type stars: since $M_{s} \propto B T^{1 / 2} / n_{\mathrm{H}}$ in the photosphere it follows that $\mathrm{d} M_{s} / \mathrm{d} z>0 \Leftrightarrow n_{\mathrm{H}} / T^{1 / 2}$ decreases with height more rapidly than $B$. It is proposed that $\mathrm{d} M_{s} / \mathrm{d} z>0$ in the photospheres of all solar type stars. For the Sun, the magnetization of the charged particles occurs over a height range of a few hundred kilometers centered around the temperature minimum of each magnetic structure.

The increasing magnetization causes the magnitude of the electric field that drives Pedersen current to increase: the increase in the magnetizations with height causes $\sigma_{\mathrm{P}}$ to decrease with height. The corresponding increase in resistance to current flow orthogonal to $\boldsymbol{B}$ causes $E_{\perp}^{\prime}$ to increase with height. This increase is essentially due to the increase of $E_{\mathrm{c}}$, which, since $B$ decreases with height, is due to the increase of $V_{\perp}$ with height.
The increasing magnetization simultaneously maximizes the efficiency of Pedersen current dissipation: the increase in the magnetizations with height causes $\sigma_{\mathrm{H}} / \sigma_{\mathrm{P}}$ to decrease with height. This causes the direction of $\boldsymbol{J}_{\perp}$ to change from being nearly orthogonal to $\boldsymbol{E}_{\perp}^{\prime}$ to being nearly parallel to it, maximizing the rate of Pedersen current dissipation for a given $\boldsymbol{J}_{\perp}$. This means that the efficiency $Q \equiv q_{\mathrm{P}} / J_{\perp} E_{\perp}^{\prime} \sim 1$. In addition, the conditions $E_{\mathrm{c}} \gg E_{\perp}$ and $q_{\mathrm{P}} \gg q_{\|}$now hold. The convection electric field becomes the dominant component of the driving electric field, and parallel current dissipation becomes insignificant. The heating rate per unit volume then reaches its global maximum value, and a temperature inversion occurs near this height.

Dissipation of metallic ion Pedersen current initiates the heating, which is rapidly dominated and maintained by dissipation of proton Pedersen current: with increasing height from the lower photosphere the temperature initially decreases approximately as for an atmosphere in radiative equilibrium, causing electron-proton recombination. The atmosphere then becomes an electron-metallic ion plasma embedded as a trace component in an $\mathrm{H}$ and $\mathrm{He} \mathrm{I}$ gas. As the electrons and ions become magnetized, heating by dissipation of ion Pedersen currents heats the atmosphere, causing $\mathrm{H}$ ionization. Due to the relatively large mass, gravitational settling, and trace concentration of the ions, the ion number density decreases rapidly with height, causing a decrease in the ion Pedersen current and associated heating rate. However, the degree of $\mathrm{H}$ ionization increases exponentially fast with increasing temperature. The protons resulting from this process rapidly dominate the Pedersen current, maintaining a heating rate large enough to cause the average thermal energy per particle, and hence the temperature to increase steadily with height as the total number density decreases with height.

It is proposed that this is the basic process by which the temperature inversion and associated chromosphere is created in the atmospheres of solar type stars.

A stellar atmosphere in which a time dependent transformation takes place between a state in which $M_{s} \ll 1$, in which case there is no chromosphere, and a state in which $M_{s} \gtrsim 1$, in which case a chromosphere is generated, may have an intermittent chromosphere. The time variation of the intermittency may be controlled by the time variation of the dynamo generated magnetic field, and atmospheric convection.

Acknowledgements. This work was supported by NSF grant ATM 0242820 to the Institute for Scientific Research. The author thanks Eugene Avrett and Rudolf Loeser of the Harvard-Smithsonian Center for Astrophysics for providing numerical solutions to the FAL models, and Juan Fontenla of the Laboratory for Space and Atmospheric Physics at the University of Colorado, Boulder for discussions about these models.

\section{References}

Anderson, L. S., \& Athay, R. G. 1989a, ApJ, 336, 1089

Anderson, L. S., \& Athay, R. G. 1989b, ApJ, 346, 1010

Avrett, E. H., \& Loeser, R. 2001, private communication 
Domínguez Cerdeña, I., Kneer, F., \& Sánchez Almeida, J. 2003, ApJ, Sánchez Almeida, J., Domínguez Cerdeña, I., \& Kneer, F. 2003, ApJ, 582, L55

Fontenla, J. M., Avrett, E. H., \& Loeser, R. 2002, ApJ, 572, 636

Goodman, M. L. 2000, ApJ, 533, 501

Goodman, M. L. 2001, Space Sci. Rev., 95, 79

Goodman, M. L. 2004, A\&A, 416, 1159

Judge, P. G., Carlsson, M., \& Stein, R. F. 2003, ApJ, 597, 1158

Lites, B. W., Rutten, R. J., \& Berger, T. E. 1999, ApJ, 517, 1013

Mitchner, M., \& Kruger, C. H. 1973, Partially ionized gases (New York: Wiley)

Sánchez Almeida, J. 1997, ApJ, 491, 993

Sánchez Almeida, J. 2000, ApJ, 544, 1135 597, L177

Sánchez Almeida, J., \& Landi degl'Innocenti, E. 1996, Sol. Phys., 164,203

Sánchez Almeida, J., Landi degl'Innocenti, E., Martinez Pillet, V., \& Lites, B. W. 1996, ApJ, 466, 537

Sánchez Almeida, J., \& Lites, B. W. 2000, ApJ, 532, 1215

Sánchez Almeida, J., Asensio Ramos, A., Trujillo Bueno, J., \& Cernicharo, J. 2001, ApJ, 555, 978

Socas-Navarro, H., \& Sánchez Almeida, J. 2002, ApJ, 565, 1323

Socas-Navarro, H., \& Sánchez Almeida, J. 2003, ApJ, 593, 581 